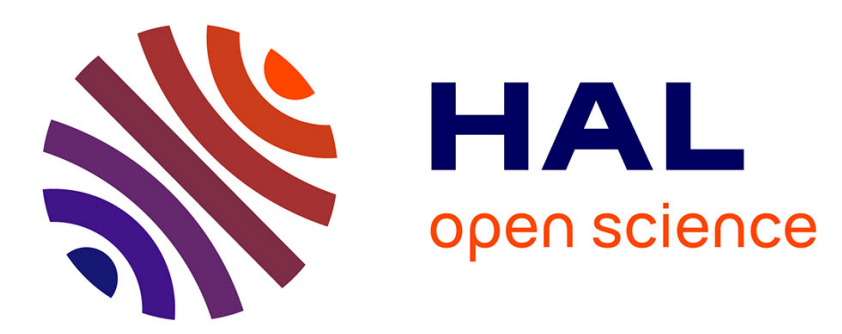

\title{
Wave Propagation in Random Waveguides with Long-Range Correlations
}

\author{
Christophe Gomez, Knut Sølna
}

\section{To cite this version:}

Christophe Gomez, Knut Sølna. Wave Propagation in Random Waveguides with LongRange Correlations. Communications in Mathematical Sciences, 2018, 16 (6), pp.1557-1596. 10.4310/CMS.2018.v16.n6.a4 . hal-01132350v3

\section{HAL Id: hal-01132350 \\ https://hal.science/hal-01132350v3}

Submitted on 9 May 2015

HAL is a multi-disciplinary open access archive for the deposit and dissemination of scientific research documents, whether they are published or not. The documents may come from teaching and research institutions in France or abroad, or from public or private research centers.
L'archive ouverte pluridisciplinaire HAL, est destinée au dépôt et à la diffusion de documents scientifiques de niveau recherche, publiés ou non, émanant des établissements d'enseignement et de recherche français ou étrangers, des laboratoires publics ou privés. 


\title{
Wave Propagation in Random Waveguides with Long-Range Correlations.
}

\author{
Christophe Gomez* and Knut Sølna ${ }^{\dagger}$
}

\begin{abstract}
In this paper we consider an acoustic waveguide with random fluctuations of its sound speed profile. These random perturbations are assumed to have long-range correlation properties. In waveguides a monochromatic wave can be decomposed in propagating modes and evanescent modes, and the random perturbation couples all these modes. Using asymptotic analysis of the modecoupling mechanism we give a description of the effects that the random fluctuations with long-rang statistical properties have on the pulse propagation.
\end{abstract}

Keywords. Acoustic waveguides, random media, asymptotic analysis, long-range correlations

AMS subject classification. 76B15, 35Q99, 60F05

\section{Introduction}

Analysis of physical measurements shows that for waves the medium of propagation may exhibit perturbations with long-range dependancies and this has stimulated interest in a mathematical understanding of how waves propagate through multiscale media $[6,16,19,20]$. Multiscale random media with longrange correlations are used to model for instance the heterogenous earth crust, the turbulent atmosphere, and also for biological tissues. There is a large literature about propagation in heterogeneous media which vary on a well defined microscale [9], but for multiscale random media the theory is not complete. In order to be efficient, imaging and communication algorithms require insight about how the wave is affected by the rough medium fluctuations. In view of its potential for applications, mathematical description of wave propagation in multiscale random media with long-range correlations has attracted a lot of interest over the last decade $[3,11,14,15,17,18,21]$.

Multiscale random medium with long-range correlations produce stochastic effects on the waves which are very different from the ones produced by perturbations varying on a well defined microscale and with mixing properties [9]. Wave propagation in random media with long-range correlations has already been considered in one-dimensional propagation media $[11,18]$ or open media under the paraxial approximation $[3,7,8,14,15]$. In these contexts, it has been observed that stochastic effects appear at different propagation scales, all the stochastic effects do not appear at the same time, which is in contrast with perturbations with mixing properties. Perturbations with long-range correlations induce first a phase modulation on the waves, a modularion that is driven by a single standard fractional Brownian motion, which does not depend on the frequency band $[3,18]$. For larger propagation distances, the random phase modulation starts to oscillate very fast to produce anomalous diffusion phenomena $[11,14,15]$. Here, we follow this line of research by considering the full wave equation in a waveguide with a continuous multiscale medium modeled through a stochastic process with long-range correlations.

In this paper, we consider acoustic wave propagation in a planar waveguide with a bounded crosssection $\mathcal{D}=(0, d)$, and given by the linearized conservation equations of mass and momentum (see [9])

$$
\begin{array}{r}
\frac{1}{K(z, x)} \partial_{t} p+\nabla \cdot \mathbf{u}=0, \\
\rho(z, x) \partial_{t} \mathbf{u}+\nabla p=\mathbf{F} .
\end{array}
$$

*Aix Marseille Université, CNRS, Centrale Marseille, I2M, UMR 7373, 13453 Marseille, France, christophe.gomez@univamu.fr.

${ }^{\dagger}$ Department of Mathematics, University of California at Irvine, Irvine, CA, USA, ksolna@math.uci.edu 
Here, $p$ is the acoustic pressure, $\mathbf{u}$ is the acoustic velocity, $\rho$ is the density of the medium, and $K$ is the bulk modulus. The coordinate $z$ represents the propagation axis along the waveguide, and the coordinate $x$ represents the bounded transverse section $\mathcal{D}=(0, d)$ of the waveguide. The forcing term $\mathbf{F}(t, z, x)$ is given by

$$
\mathbf{F}(t, z, x)=f(t) \Psi(x) \delta\left(z-L_{S}\right) \mathbf{e}_{z},
$$

where $\mathbf{e}_{z}$ is the unit vector pointing in the $z$-direction. Therefore, this term models a source located in the plan $z=L_{S}<0$, emitting a wave $f(t)$ in the $z$-direction, with a transverse profile $\Psi(x)$ (see Figure 1). In this paper, we assume that the medium parameters are given by :

$$
\begin{aligned}
\frac{1}{K(z, x)} & =\left\{\begin{array}{cll}
\frac{1}{K}(1+\sqrt{\varepsilon} V(z, x)) & \text { if } \quad z \in\left[0, L / \varepsilon^{s}\right] \\
\frac{1}{K} & \text { if } \quad z \in(-\infty, 0) \cup\left(L / \varepsilon^{s},+\infty\right)
\end{array} \quad \text { and } \quad x \in(0, d),\right. \\
\rho(z, x) & =\rho, \quad z \in(-\infty,+\infty) \text { and } x \in(0, d),
\end{aligned}
$$

where $V(z, x)$ models the fluctuations of the propagation medium, and the parameter $s$ describes the order of magnitude with respect to $\varepsilon$ of the propagation distance in the random section. This last parameter will be chosen so that we observe nontrivial stochastic effects. The medium perturbations $V(z, x)$ are assumed to be given by

$$
V(z, x)=\Theta\left(\mathcal{B}_{\mathfrak{h}}(z, x)\right),
$$

where $\mathcal{B}_{\mathfrak{h}}$ is a mean-zero continuous Gaussian random field with covariance function

$$
\mathbb{E}\left[\mathcal{B}_{\mathfrak{h}}(z+s, x) \mathcal{B}_{\mathfrak{h}}(s, y)\right]=r_{\mathfrak{h}}(z) R(x, y),
$$

with $r_{\mathfrak{h}}(0)=R(x, x)=1$, and such that

$$
r_{\mathfrak{h}}(z) \underset{z \rightarrow+\infty}{\sim} \frac{c_{\mathfrak{h}}}{z^{\mathfrak{h}}} \quad \text { with } \quad \mathfrak{h} \in(0,1) .
$$

This last relation is the so-called long-range property. The function $\Theta$ is a bounded smooth and odd function on $\mathbb{R}$ so that the bulk modulus $K$ takes only positive values and $\mathbb{E}[V(z, x)]=0$. However, for technical reasons in the proof of the main result we need the following assumption on $\Theta$. In this paper, we assume that for all $n \geq 1$

$$
\sum_{l \geq 1} \frac{\left|\Theta_{l}\right|}{\sqrt{l !}}(n-1)^{l / 2}<+\infty
$$

with

$$
\Theta_{l}:=\left\langle H_{l}, \Theta\right\rangle_{L^{2}(\mathbb{R}, w(u) d u)},
$$

where $\left(H_{l}\right)_{l \geq 1}$ are the Hermite polynomials defined by

$$
H_{l}(u):=(-1)^{l} \frac{w^{(l)}(u)}{w(u)}, \quad \text { with } \quad w(u):=\frac{e^{-u^{2} / 2}}{\sqrt{2 \pi}} .
$$

Let us remark that (5) is satisfies if $\Theta$ is a polynomial. Finally, we can also show (see Proposition 5.1 Section 5) the long-range property for $V$, which is

$$
\mathbb{E}[V(z+s, x) V(s, y)] \underset{z \rightarrow+\infty}{\sim} \frac{C_{\mathfrak{h}}}{z^{\mathfrak{h}}} R(x, y) \quad \text { with } \quad C_{\mathfrak{h}}=\frac{c_{\mathfrak{h}}}{2 \pi}\left(\int_{-\infty}^{+\infty} x \Theta(x) e^{-x^{2} / 2} d x\right)^{2} .
$$

The main consequence of the long-range property on the medium perturbations is that its autocorrelation function is not integrable,

$$
\int_{0}^{+\infty}|\mathbb{E}[V(z+s, x) V(s, y)]| d z=+\infty,
$$

and this is the reason why the cumulative stochastic effects on the wave propagation are strongly different with respect to the ones obtained in classical mixing case (see [9, Chapter 20] and [11, 18, 15]).

The goal of this paper is to prove that waves propagating in the random waveguide with $s=1 /(2-\mathfrak{h})$ exhibit a mode- and frequency-dependent random phase modulations. However, for all the frequencies generated by the source and all the propagating modes of the wave decomposition the randomness of the phase modulations is defined in terms of the same standard fractional Brownian motion. This result is consistent with the ones already obtained in $[3,18]$ for perturbations with long-range correlations, 


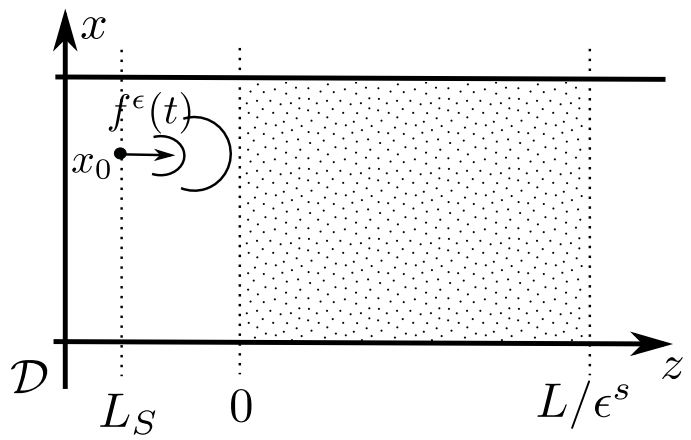

Figure 1: Illustration of the waveguide model. The source $f^{\varepsilon}$ generates a wave that is propagating in the positive $z$ direction. The section $z \in\left[0, L / \varepsilon^{s}\right]$ is randomly heterogeneous with long range correlations. Our objective is to characterize the pulse as it emerges on the termination of the random section at $z=L / \varepsilon^{s}$.

and in contrast with the ones obtained for perturbations with mixing properties. In fact, in the latter case the phase modulations is obtain for $s=1$ and are given by a vector of Brownian motions. The size of this vector depends on the number of propagating modes, and its correlation matrix depends on the frequency band of the source [9, Chapter 20]. To prove the main result of this paper, we use a moment technique [3] which is very convenient for long-range correlations with Gaussian underlying fluctuations.

The organization of this paper is as follows. In Section 1, we describe the wave propagation in waveguides through a modal decomposition, and the mode-coupling mechanism induced by the medium perturbations. In Section 2, we state the main result of this paper, which is used in Section 3 and Section 4 to study the pulse propagation. In Section 5 we give technical results to handle the long-range dependencies of the medium perturbations. These are used in Section 6 to prove the main result of this paper (Theorem 2.1). Finally, in Section 7 and Section 8, we prove respectively Theorem 3.1 and Theorem 3.2 stated in Section 3.

\section{Wave Propagation}

In this section we describe the general strategy to study wave propagation in waveguides. From (1) we obtain the standard wave equation for the pressure wave :

$$
\Delta p-\frac{1}{c^{2}}\left(1+\sqrt{\varepsilon} V(z, x) \mathbf{1}_{\left[0, L / \varepsilon^{s}\right]}(z)\right) \partial_{t}^{2} p=\nabla \cdot \mathbf{F},
$$

where $\Delta=\partial_{z}^{2}+\partial_{x}^{2}$ and $c=\sqrt{K / \rho}$ is the sound speed. In this paper we consider Dirichlet boundary conditions (Neumann boundary conditions are considered in Section 4)

$$
p(t, 0, z)=p(t, d, z)=0, \quad \forall(t, z) \in \mathbb{R} \times \mathbb{R} .
$$

The wave equation (7) is a linear equation so that the pressure wave can be decomposed as a superposition of monochromatic wave with the Fourier transform

$$
\widehat{p}(\omega, x, z)=\int p(t, x, z) e^{i \omega t} d t \quad \text { and } \quad p(t, x, z)=\frac{1}{2 \pi} \int \widehat{p}(\omega, x, z) e^{-i \omega t} d t .
$$

Therefore, the pressure field $\widehat{p}(\omega, x, z)$ satisfies the following Helmholtz equation (time-harmonic wave equation) in $z \in\left(L_{S},+\infty\right)$ (resp. $z \in\left(-\infty, L_{S}\right)$ )

$$
\partial_{z}^{2} \widehat{p}(\omega, x, z)+\partial_{x}^{2} \widehat{p}(\omega, x, z)+k^{2}(\omega)\left(1+\sqrt{\varepsilon} V(z, x) \mathbf{1}_{\left[0, L / \varepsilon^{s}\right]}(z)\right) \widehat{p}(\omega, x, z)=0,
$$

where $k(\omega)=\omega / c$ is the wavenumber, and with Dirichlet boundary conditions,

$$
\widehat{p}(\omega, 0, z)=\widehat{p}(\omega, d, z)=0, \quad \forall z \in \mathbb{R} .
$$


Moreover, according to the shape of the source term (2) the pressure field satisfies the following jump conditions and longitudinal velocity continuity across the plane $z=L_{S}$

$$
\widehat{p}\left(\omega, x, L_{S}^{+}\right)-\widehat{p}\left(\omega, x, L_{S}^{-}\right)=\widehat{f^{\varepsilon}}(\omega) \Psi(x) \quad \text { and } \quad \partial_{z} \widehat{p}\left(\omega, x, L_{S}^{+}\right)=\partial_{z} \widehat{p}\left(\omega, x, L_{S}^{-}\right)
$$

The transverse Laplacian $-\partial_{x}^{2}$ with Dirichlet boundary conditions on $\partial \mathcal{D}$ is a positive self-adjoint operator in $L^{2}(\mathcal{D})$, and then its spectrum is composed of a countably infinite number of positive eigenvalues $\left(\lambda_{j}\right)_{j \geq 1}$ since $\mathcal{D}=(0, d)$ is a bounded domain. Therefore, let us introduce for all $j \geq 1$

$$
-\partial_{x}^{2} \phi_{j}(x)=\lambda_{j} \phi_{j}(x) \quad \forall x \in \mathcal{D}, \quad \text { and } \quad \phi_{j}(0)=\phi_{j}(d)=0,
$$

where $0<\lambda_{1}<\lambda_{2}<\cdots$ and the eigenvectors $\left(\phi_{j}\right)_{j \geq 1}$ form an orthonormal basis of $L^{2}(\mathcal{D})$,

$$
\int_{0}^{d} \phi_{j}(x) \phi_{l}(x) d x=\delta_{j l} .
$$

Here, $\delta_{j l}$ denotes the Kronecker symbol. In the planar case $\mathcal{D}=(0, d)$, we have explicit expressions for the eigenvectors and eigenvalues :

$$
\phi_{j}(x)=\sqrt{\frac{2}{d}} \sin (j \pi x / d) \quad \text { and } \quad \lambda_{j}=\frac{j^{2} \pi^{2}}{d^{2}} \quad \forall j \geq 1 .
$$

As a result, we have the following decomposition of the wave field

$$
\widehat{p}(\omega, x, z)=\sum_{j \geq 1} \widehat{p}_{j}(\omega, z) \phi_{j}(x)
$$

The two next sections present the modal decomposition of $\widehat{p}(\omega, x, z)$, first in a homogeneous waveguide $(V \equiv 0)$ and then for the randomly perturbed waveguide.

\subsection{Modal Decomposition in Homogeneous Waveguides $(V \equiv 0)$}

This section is devoted to the modal decomposition (10) for a homogeneous waveguide. This describes the wave propagation from the source location $z=L_{S}$ to the beginning of the random section $z=0$ (see Figure 1). According to (8) and (10), we have for all $z \neq L_{S}$ and $j \geq 1$

$$
\frac{d^{2}}{d z^{2}} \widehat{p}_{j}(\omega, z)+\beta_{j}^{2}(\omega) \widehat{p}_{j}(\omega, z)=0
$$

with

$$
\beta_{j}(\omega)=\sqrt{k^{2}(\omega)-\lambda_{j}}, \quad \text { for } j \in\{1, \ldots, N(\omega)\}
$$

and

$$
\beta_{j}(\omega)=\sqrt{\lambda_{j}-k^{2}(\omega)}, \quad \text { for } j \geq N(\omega)+1
$$

Here, $N(\omega)$ is the integer such that $\lambda_{N(\omega)} \leq k^{2}(\omega)<\lambda_{N(\omega)+1}$, that is for our planar waveguide

$$
N(\omega)=\left[\frac{\omega d}{\pi c}\right]
$$

where [.] stands for the integer part. As a result, according to (11) in addition to (9), the pressure field can be expended as follows

$$
\begin{aligned}
\widehat{p}(\omega, x, z) & =\left[\sum_{j=1}^{N(\omega)} \frac{\widehat{a}_{j, 0}(\omega)}{\sqrt{\beta_{j}(\omega)}} e^{i \beta_{j}(\omega) z} \phi_{j}(x)+\sum_{j \geq N(\omega)+1} \widehat{p}_{j, 0}(\omega) e^{-\beta_{j}(\omega) z} \phi_{j}(x)\right] \mathbf{1}_{\left(L_{S},+\infty\right)}(z) \\
& +\underbrace{\left[\sum_{j=1}^{N(\omega)} \frac{\widehat{b}_{j, 0}(\omega)}{\sqrt{\beta_{j}(\omega)}} e^{-i \beta_{j}(\omega) z} \phi_{j}(x)\right.}_{\text {propagating modes }}+\underbrace{\left.\sum_{j \geq N(\omega)+1} \widehat{q}_{j, 0}(\omega) e^{\beta_{j}(\omega) z} \phi_{j}(x)\right]}_{\text {evanescent modes }} \mathbf{1}_{\left(-\infty, L_{S}\right)}(z),
\end{aligned}
$$




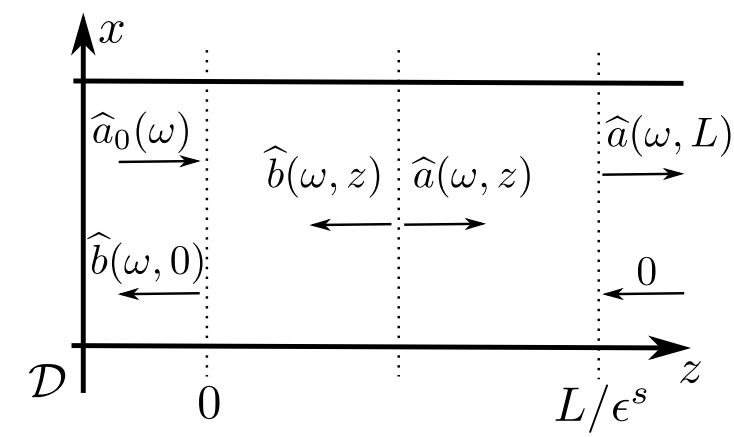

Figure 2: Illustration of the right-going and left-going propagating mode amplitudes $\widehat{a}(\omega, z)$ and $\widehat{b}(\omega, z)$. The source generate the probing wave $\widehat{a}_{0}(\omega)$, the reflected wave is $\widehat{b}(\omega, 0)$ and the transmitted wave, which is our interest is $\widehat{a}\left(\omega, L / \varepsilon^{s}\right)$. Note that there is no energy coming from $z>L / \varepsilon^{s}$.

with

$$
\widehat{a}_{j, 0}(\omega)=-\widehat{b}_{j, 0}(\omega)=\frac{\sqrt{\beta_{j}(\omega)}}{2} \widehat{f}(\omega) e^{-i \beta_{j}(\omega) L_{S}}\left\langle\phi_{j}, \Psi\right\rangle_{L^{2}(0, d)}, \quad \text { for } j \in\{1, \ldots, N(\omega)\},
$$

and

$$
\widehat{p}_{j, 0}(\omega) e^{-\beta_{j}(\omega) L_{S}}=-\widehat{q}_{j, 0}(\omega) e^{\beta_{j}(\omega) L_{S}}=\frac{\sqrt{\beta_{j}(\omega)}}{2} \widehat{f}(\omega)\left\langle\phi_{j}, \Psi\right\rangle_{L^{2}(0, d)}, \quad \text { for } j \geq N(\omega)+1 .
$$

In (12), we refer to the modes with $j \in\{1, \ldots, N(\omega)\}$ as propagating modes, these are modes which can propagate over large distances. The evanescent mode are the modes with $j \geq N(\omega)+1$, and correspond to modes which cannot propagate over large distance. Here, $N(\omega)$ corresponds to the number of propagating modes, $\widehat{a}_{j, 0}(\omega)$ (resp. $\widehat{b}_{j, 0}(\omega)$ ) is the amplitude of the $j$ th right-going (resp. left-going) propagating mode, and $\widehat{p}_{j, 0}(\omega)$ (resp. $\widehat{q}_{j, 0}(\omega)$ ) is the amplitude of the $j$ th right-going (resp. left-going) evanescent mode.

\subsection{Mode Coupling for Randomly Perturbed Waveguides}

In this section we are interested in the modal decomposition of the pressure field over the randomly perturbed section of the waveguide $\left(0, L / \varepsilon^{s}\right)$. In this case, the perturbations of the propagation medium induce a mode coupling. To describe this coupling mechanism, let us introduce the right-going and left-going propagating mode amplitudes $\widehat{a}_{j}(\omega, z)$ and $\widehat{b}_{j}(\omega, z)(j \in\{1, \ldots, N(\omega)\})$ such that

$$
\widehat{p}_{j}(z)=\frac{\widehat{a}_{j}(z) e^{i \beta_{j} z}+\widehat{b}_{j}(z) e^{-i \beta_{j} z}}{\sqrt{\beta_{j}}} \text { and } \partial_{z} \widehat{p}_{j}(z)=i \sqrt{\beta_{j}}\left(\widehat{a}_{j}(z) e^{i \beta_{j} z}-\widehat{b}_{j}(z) e^{-i \beta_{j} z}\right)
$$

(see Figure 2) which are defined by

$$
\widehat{a}_{j}(z)=\frac{i \beta_{j} \widehat{p}_{j}(z)+\partial_{z} \widehat{p}_{j}(z)}{2 i \sqrt{\beta_{j}}} e^{-i \beta_{j} z} \quad \text { and } \quad \widehat{b}_{j}(z)=\frac{i \beta_{j} \widehat{p}_{j}(z)-\partial_{z} \widehat{p}_{j}(z)}{2 i \sqrt{\beta_{j}}} e^{i \beta_{j} z} .
$$

Therefore, according to (8) and (10), we have the following coupled differential equations for the mode amplitudes

$$
\begin{aligned}
\frac{d}{d z} \widehat{a}_{j}(\omega, z) & =\sqrt{\varepsilon} \frac{i k^{2}(\omega)}{2} \sum_{l=1}^{N(\omega)} C_{j l}(\omega, z)\left(\widehat{a}_{j}(\omega, z) e^{i\left(\beta_{l}(\omega)-\beta_{j}(\omega)\right) z}+\widehat{b}_{j}(\omega, z) e^{-i\left(\beta_{l}(\omega)+\beta_{j}(\omega)\right) z}\right) \\
& +\sqrt{\varepsilon} \frac{i k^{2}(\omega)}{2} \sum_{l \geq N(\omega)+1} C_{j l}(\omega, z) \sqrt{\beta_{l}(\omega)} \widehat{p}_{l}(\omega, z) e^{-i \beta_{j}(\omega) z},
\end{aligned}
$$




$$
\begin{aligned}
\frac{d}{d z} \widehat{b}_{j}(\omega, z) & =-\sqrt{\varepsilon} \frac{i k^{2}(\omega)}{2} \sum_{l=1}^{N(\omega)} C_{j l}(\omega, z)\left(\widehat{a}_{j}(\omega, z) e^{i\left(\beta_{l}(\omega)+\beta_{j}(\omega)\right) z}+\widehat{b}_{j}(\omega, z) e^{-i\left(\beta_{l}(\omega)-\beta_{j}(\omega)\right) z}\right) \\
& -\sqrt{\varepsilon} \frac{i k^{2}(\omega)}{2} \sum_{l \geq N(\omega)+1} C_{j l}(\omega, z) \sqrt{\beta_{l}(\omega)} \widehat{p}_{l}(\omega, z) e^{i \beta_{j}(\omega) z},
\end{aligned}
$$

for $j \in\{1, \ldots, N(\omega)\}$, and

$$
\frac{d^{2}}{d z^{2}} \widehat{p}_{j}(\omega, z)-\beta_{j}^{2}(\omega) \widehat{p}_{j}(\omega, z)+2 \sqrt{\varepsilon} g_{j}(\omega, z)=0,
$$

for $j \geq N(\omega)+1$ where

$$
g_{j}(z)=k^{2} \sum_{l \geq N+1} C_{j l}(z) \sqrt{\beta_{j} \beta_{l}} \widehat{p}_{l}(z)+k^{2} \sum_{l=1}^{N} C_{j l}(z) \sqrt{\beta_{j}}\left(\widehat{a}_{l}(z) e^{i \beta_{l} z}+\widehat{b}_{l}(z) e^{-i \beta_{l} z}\right) .
$$

In these equations the coupling coefficients are defined by

$$
C_{j l}(z)=\frac{1}{\sqrt{\beta_{j} \beta_{l}}} \int_{\mathcal{D}} V(z, x) \phi_{j}(x) \phi_{l}(x) d x .
$$

We complement the systems (14), (15) and (16) with the boundary conditions

$$
\widehat{a}(\omega, 0)=\widehat{a}_{0}(\omega) \quad \text { and } \quad \widehat{b}\left(\omega, L / \varepsilon^{s}\right)=0,
$$

(see Figure 2) where $\widehat{a}_{0}(\omega)$ is defined by (13). The second condition means that no wave is incoming from the right hand side of the perturbed section $\left(0, L / \varepsilon^{s}\right)$. We also introduce the following radiation condition

$$
\lim _{z \rightarrow+\infty} \sum_{l \geq N(\omega)+1}\left|\widehat{p}_{j}(\omega, z)\right|^{2}=0
$$

meaning that the energy carried by the evanescent modes decay to 0 at infinity. Using this condition, one can show following [10, Section 3.2] the global conservation relation

$$
\left\|\widehat{a}\left(\omega, L / \varepsilon^{s}\right)\right\|_{\mathbb{C}^{N(\omega)}}^{2}+\|\widehat{b}(\omega, 0)\|_{\mathbb{C}^{N(\omega)}}^{2}=\left\|\widehat{a}_{0}(\omega)\right\|_{\mathbb{C}^{N(\omega)}}^{2},
$$

where $\|\cdot\|_{\mathbb{C}^{N(\omega)}}$ stands for the Euclidian norm on $\mathbb{C}^{N(\omega)}$.

\subsection{Propagating Mode Amplitude Equations and Propagator}

The coupled mode equations (14) and (15) for the propagating mode amplitudes are not closed, they involve also the evanescent mode amplitudes. However, it is possible to derive a closed system (21) for the propagating mode amplitudes taking into account the influence of the evanescent modes on the propagating modes up to an error leading to negligible effects compared to the ones discussed in the remaining of the paper (see $[9,10,12,13])$. From now on, let us consider the following coupled differential equations for the mode amplitudes

$$
\frac{d}{d z}\left[\begin{array}{l}
\widehat{a}(\omega, z) \\
\widehat{b}(\omega, z)
\end{array}\right]=[\sqrt{\varepsilon} \mathbf{H}(\omega, z)+\varepsilon \mathbf{G}(\omega, z)]\left[\begin{array}{l}
\widehat{a}(\omega, z) \\
\widehat{b}(\omega, z)
\end{array}\right]
$$

with boundary conditions given by (18), and where

$$
\mathbf{H}(\omega, z)=\left[\begin{array}{ll}
\mathbf{H}^{a}(\omega, z) & \overline{\mathbf{H}^{b}(\omega, z)} \\
\mathbf{H}^{b}(\omega, z) & \overline{\mathbf{H}^{a}(\omega, z)}
\end{array}\right] \quad \text { and } \quad \mathbf{G}(\omega, z)=\left[\begin{array}{ll}
\mathbf{G}^{a}(\omega, z) & \overline{\mathbf{G}^{b}(\omega, z)} \\
\mathbf{G}^{b}(\omega, z) & \overline{\mathbf{G}^{a}(\omega, z)}
\end{array}\right]
$$

with

$$
\begin{aligned}
& \mathbf{H}_{j l}^{a}(z)=\frac{i k^{2}}{2} C_{j l}(z) e^{i\left(\beta_{l}-\beta_{j}\right) z}, \quad \mathbf{H}_{j l}^{b}(z)=-\frac{i k^{2}}{2} C_{j l}(z) e^{i\left(\beta_{l}+\beta_{j}\right) z} \\
& \mathbf{G}_{j l}^{a}(z)=\frac{i k^{4}}{4} \sum_{l^{\prime} \geq N+1} \int_{-\infty}^{+\infty} C_{j l^{\prime}}(z) C_{l^{\prime} l}(z+u) e^{i \beta_{l}(z+u)-i \beta_{j} z-\beta_{l^{\prime}}|u|} d u, \\
& \mathbf{G}_{j l}^{b}(z)=-\frac{i k^{4}}{4} \sum_{l^{\prime} \geq N+1} \int_{-\infty}^{+\infty} C_{j l^{\prime}}(z) C_{l^{\prime} l}(z+u) e^{i \beta_{l}(z+u)+i \beta_{j} z-\beta_{l^{\prime}}|u|} d u
\end{aligned}
$$


and $C_{j l}(z)$ defined by (17). Here, the matrices $\mathbf{H}^{a}$ and $\mathbf{H}^{b}$ describe the coupling between the propagating modes, while the matrices $\mathbf{G}^{a}$ and $\mathbf{G}^{b}$ describe the coupling between the evanescent modes with the propagating modes. Rescaling the propagating mode amplitudes according to the order of magnitude of the propagation distance, we consider

$$
\widehat{a}^{\varepsilon}(\omega, z)=\widehat{a}\left(\omega, z / \varepsilon^{s}\right) \quad \text { and } \quad \widehat{b}^{\varepsilon}(\omega, z)=\widehat{b}\left(\omega, z / \varepsilon^{s}\right),
$$

for $z \in[0, L]$, satisfying the scaled coupled mode equations

$$
\frac{d}{d z}\left[\begin{array}{l}
\widehat{a}^{\varepsilon}(\omega, z) \\
\widehat{b}^{\varepsilon}(\omega, z)
\end{array}\right]=\left[\frac{1}{\varepsilon^{s-1 / 2}} \mathbf{H}\left(\omega, \frac{z}{\varepsilon^{s}}\right)+\varepsilon^{1-s} \mathbf{G}\left(\omega, \frac{z}{\varepsilon^{s}}\right)\right]\left[\begin{array}{l}
\widehat{a}^{\varepsilon}(\omega, z) \\
\widehat{b}^{\varepsilon}(\omega, z)
\end{array}\right]
$$

with boundary conditions

$$
\widehat{a}^{\varepsilon}(\omega, 0)=\widehat{a}_{0}(\omega) \quad \text { and } \quad \widehat{b}^{\varepsilon}(\omega, L)=0 .
$$

The two-point boundary value problem (23) and (24) can be solved using the propagator matrix defined as the unique solution of

$$
\frac{d}{d z} \mathbf{P}^{\varepsilon}(\omega, z)=\left[\frac{1}{\varepsilon^{s-1 / 2}} \mathbf{H}\left(\omega, \frac{z}{\varepsilon^{s}}\right)+\varepsilon^{1-s} \mathbf{G}\left(\omega, \frac{z}{\varepsilon^{s}}\right)\right] \mathbf{P}^{\varepsilon}(\omega, z) \quad \text { with } \quad \mathbf{P}^{\varepsilon}(\omega, 0)=I d_{2 N(\omega)},
$$

so that for all $z \in[0, L]$

$$
\left[\begin{array}{l}
\widehat{a}^{\varepsilon}(\omega, z) \\
\widehat{b}^{\varepsilon}(\omega, z)
\end{array}\right]=\mathbf{P}^{\varepsilon}(\omega, z)\left[\begin{array}{l}
\widehat{a}^{\varepsilon}(\omega, 0) \\
\widehat{b}^{\varepsilon}(\omega, 0)
\end{array}\right] \quad \text { and } \quad\left[\begin{array}{c}
\widehat{a}^{\varepsilon}(\omega, L) \\
0
\end{array}\right]=\mathbf{P}^{\varepsilon}(\omega, L)\left[\begin{array}{l}
\widehat{a}_{0}(\omega, 0) \\
\widehat{b}^{\varepsilon}(\omega, 0)
\end{array}\right]
$$

according to (18). Because of the form of $\mathbf{H}(\omega, z)$ and $\mathbf{G}(\omega, z)$ the propagator can be expressed as

$$
\mathbf{P}^{\varepsilon}(\omega, z)=\left[\begin{array}{ll}
\mathbf{P}^{a, \varepsilon}(\omega, z) & \overline{\mathbf{P}^{b, \varepsilon}(\omega, z)} \\
\mathbf{P}^{b, \varepsilon}(\omega, z) & \overline{\mathbf{P}^{a, \varepsilon}(\omega, z)}
\end{array}\right]
$$

where $\mathbf{P}^{a, \varepsilon}(\omega, z)$ describes the coupling mechanisms between the right-going modes (resp. left-going modes) with themselves, while $\mathbf{P}^{b, \varepsilon}(\omega, z)$ describes the coupling mechanisms between the right-going and left-going modes.

In the next section we study the asymptotic distribution as $\varepsilon \rightarrow 0$ of the propagator and we specify the propagation parameter $s$ leading to a nontrivial asymptotic behavior.

\section{Phase Modulation for the Propagator}

This section is devoted to the main results of this paper, which are used to describe the pulse propagation in the two next sections. In the following theorem we describe the asymptotic distribution as $\varepsilon$ goes to 0 of the couple of matrices

$$
\mathcal{P}^{\varepsilon}(\omega, z)=\left[\begin{array}{l}
\mathbf{P}^{a, \varepsilon}(\omega, z) \\
\mathbf{P}^{b, \varepsilon}(\omega, z)
\end{array}\right] \in \mathcal{M}_{N(\omega)}(\mathbb{C}) \times \mathcal{M}_{N(\omega)}(\mathbb{C})
$$

where $\mathcal{M}_{N(\omega)}(\mathbb{C})$ stands for the set of $N(\omega) \times N(\omega)$ matrices with complex coefficients.

Theorem 2.1 For $s=1 /(2-\mathfrak{h})$ and for all $z \in[0, L]$, the family $\left(\mathcal{P}^{\varepsilon}(\omega, z)\right)_{\varepsilon}$ converges in distribution in $\mathcal{M}_{N}(\mathbb{C}) \times \mathcal{M}_{N}(\mathbb{C})$ to

$$
\left[\begin{array}{c}
D(\omega, z) \\
\mathbf{0}
\end{array}\right]
$$

with

$$
D(\omega, z)=\operatorname{diag}\left(e^{i \sigma_{1, H}(\omega) B_{H}(z)}, \ldots, e^{i \sigma_{N, H}(\omega) B_{H}(z)}\right),
$$

where $B_{H}$ is a standard fractional Brownian motion with Hurst index $H=(2-\mathfrak{h}) / 2 \in(1 / 2,1)$,

$$
\sigma_{j, H}(\omega)=\frac{k^{2}(\omega)}{2 \beta_{j}(\omega)} \sqrt{\frac{C_{\mathfrak{h}} R_{j j j j}}{H(2 H-1)}}
$$

and

$$
R_{m n p q}=\iint_{\mathcal{D} \times \mathcal{D}} R\left(x, x^{\prime}\right) \phi_{m}(x) \phi_{n}(x) \phi_{p}\left(x^{\prime}\right) \phi_{q}\left(x^{\prime}\right) d x d x^{\prime}
$$


The proof of Theorem 2.1 is given in Section 6. We now present several remarks regarding this result. First, Theorem 2.1 implies that the first significant stochastic effects affecting the wave take place for $s=1 /(2 H)<1$. This is in contrast to the classical mixing case (see [9, Chapter 20]) for which all the stochastic effects appear for $s=1$. The second one concerns the convergence of $\mathbf{P}^{b, \varepsilon}(\omega, z)$ in probability to $\mathbf{0}$ meaning that the coupling mechanisms between the right-going and left-going modes are negligible for $\varepsilon$ small. In other words, the backscattering is negligible for $\varepsilon$ small. The third one concerns the convergence in distribution of $\mathbf{P}^{a, \varepsilon}(\omega, z)$ to a diagonal matrix, which means that the coupling mechanisms between two different right-going modes is also negligible for $\varepsilon$ small. Finally, the propagating modes are only affected by mode- and frequency-dependent phase modulations but driven by the same fractional Brownian motion, which does not depend on the frequency. This effect of modedependent phase modulation driven by a single fractional Brownian motion has already been observed in [3, Theorem 1.2] for the random Schrödinger equation with long-range correlations.

We finish this section with two multifrequency versions of Theorem 2.1 which will be useful for the study of the pulse propagation in the next section. The proofs of the following requires only simple modifications of the one of Theorem 2.1.

Theorem 2.2 Let $s=1 /(2-\mathfrak{h}), \gamma \in \mathbb{N}^{*}$, and $z \in[0, L]$.

- For all $\left(\omega_{1}, \ldots, \omega_{\gamma}\right)$ such that

$$
N\left(\omega_{j}\right)=N \quad \forall j \in\{1, \ldots, \gamma\},
$$

the family $\left(\mathcal{P}^{\varepsilon}\left(\omega_{1}, z\right), \ldots, \mathcal{P}^{\varepsilon}\left(\omega_{\gamma}, z\right)\right)_{\varepsilon}$ converges in distribution in $\left(\mathcal{M}_{N}(\mathbb{C}) \times \mathcal{M}_{N}(\mathbb{C})\right)^{\gamma}$ to

$$
\left[\begin{array}{ccc}
D\left(\omega_{1}, z\right) & & D\left(\omega_{\gamma}, z\right) \\
\mathbf{0} & & \mathbf{0}
\end{array}\right],
$$

- For all $M>0$, and $\left(h_{1}, \ldots, h_{\gamma}\right) \in[-M, M]^{\gamma}$, the family $\left(\mathcal{P}^{\varepsilon}\left(\omega+\varepsilon^{q} h_{1}, z\right), \ldots, \mathcal{P}^{\varepsilon}\left(\omega+\varepsilon^{q} h_{\gamma}, z\right)\right)_{\varepsilon}$, with $q \in(0, s]$, converges in distribution in $\left(\mathcal{M}_{N(\omega)}(\mathbb{C}) \times \mathcal{M}_{N(\omega)}(\mathbb{C})\right)^{\gamma}$ to

$$
\left[\begin{array}{ccc}
D(\omega, z) & & D(\omega, z) \\
\mathbf{0} & \ldots & \mathbf{0}
\end{array}\right],
$$

where $D(\omega, z)$ is defined by $(27)$.

This theorem characterize the behavior of the propagator at different and nearby frequencies. It turns out that the energy and the asymptotic statistical behavior of the propagator for different frequencies are not affected on the propagation scale $L / \varepsilon^{s}$ with $s=1 /(2-\mathfrak{h})$. On this scale the wave does not propagate enough and accumulate enough scattering events to affect the frequency coherence nor the energy. This type of phenomenon has already been observed in [11] for one-dimensional propagation media and in [15] for the random Schrödinger equation with long-range correlations.

\section{Pulse Propagation for $s=1 /(2-\mathfrak{h})$}

In this section we describe the asymptotic behavior of a pulse at the end of the random section $z=L / \varepsilon^{s}$ with $s=1 /(2-\mathfrak{h})$. We assume that the pulse has been generated by the source (2) with

$$
f^{\varepsilon}(t)=f\left(\varepsilon^{q} t\right) e^{-i \omega_{0} t}, \quad \text { so that } \quad \widehat{f}^{\varepsilon}(\omega)=\frac{1}{\varepsilon^{q}} \widehat{f}\left(\frac{\omega-\omega_{0}}{\varepsilon^{q}}\right) .
$$

This profile models a source with carrier frequency $\omega_{0}$ and bandwidth of order $\varepsilon^{q}$. However, for the sake of simplicity we assume that $\widehat{f}(\omega)$ is a compactly supported smooth function. Throughout this section, we refer to a broadband pulse if $q<s$ (the order of the pulse width is small compared to the propagation distance $\varepsilon^{-q} \ll \varepsilon^{-s}$ ) and narrowband if $q=s$ (the order of the pulse width is comparable to the order of the propagation distance $\varepsilon^{-q}=\varepsilon^{-s}$ ). According to the boundary conditions (24) (no wave is incoming from the right) the pulse can be decomposed into two parts

$$
p\left(t, x, \frac{L}{\varepsilon^{s}}\right)=\tilde{p}_{p r}\left(t, x, \frac{L}{\varepsilon^{s}}\right)+p_{e}\left(t, x, \frac{L}{\varepsilon^{s}}\right),
$$


where

$$
\tilde{p}_{p r}\left(t, x, \frac{L}{\varepsilon^{s}}\right)=\frac{1}{2 \pi} \int d \omega e^{-i \omega t} \sum_{j=1}^{N(\omega)} \frac{\widehat{a}_{j}^{\varepsilon}(\omega, L)}{\sqrt{\beta_{j}(\omega)}} e^{i \beta_{j}(\omega) L / \varepsilon^{s}} \phi_{j}(x)
$$

is the propagating part of the pulse, and

$$
p_{e}\left(t, x, \frac{L}{\varepsilon^{s}}\right)=\frac{1}{2 \pi} \int d \omega e^{-i \omega t} \sum_{j \geq N(\omega)+1} \widehat{p}_{j}\left(\omega, L / \varepsilon^{s}\right) \phi_{j}(x)
$$

is the evanescent part of the pulse, which is small in the limit $\varepsilon \rightarrow 0$. Therefore, in what follows we focus our attention on the propagating component of the pressure wave. In order to use Theorem 2.1 we need to express the forward-propagating mode amplitudes $\widehat{a}^{\varepsilon}(\omega, L)$ in terms of $\mathcal{P}^{\varepsilon}(\omega, L)$. This can be done according to the right-hand side of (26), note though that the backward-propagating mode amplitudes $\widehat{b}^{\varepsilon}(\omega, 0)$ are not specified. However, using the fact that $\mathbf{P}^{b, \varepsilon}(\omega, L)$ converges in probability to 0 and $(20)$, we have

$$
\lim _{\varepsilon \rightarrow 0} \mathbb{P}\left(\sup _{t, x}\left|p\left(t, x, L / \varepsilon^{s}\right)-p_{p r}\left(t, x, L / \varepsilon^{s}\right)\right|>\eta\right)=0
$$

where

$$
\begin{aligned}
p_{p r}\left(t, x, \frac{L}{\varepsilon^{s}}\right)=\frac{1}{4 \pi \varepsilon^{q}} \int d \omega e^{-i \omega t} \widehat{f}\left(\frac{\omega-\omega_{0}}{\varepsilon^{q}}\right) & \sum_{j, l=1}^{N(\omega)} \sqrt{\frac{\beta_{l}(\omega)}{\beta_{j}(\omega)}} \mathbf{P}_{j l}^{a, \varepsilon}(\omega, L) \\
& \times e^{i \beta_{j}(\omega) L / \varepsilon^{s}} e^{-i \beta_{l}(\omega) L_{S}} \phi_{j}(x)\left\langle\phi_{l}, \Psi\right\rangle_{L^{2}(0, d)}
\end{aligned}
$$

As a result, the asymptotic behavior of the pulse $p\left(t, x, L / \varepsilon^{s}\right)$ is equivalent to the one of $p_{p r}\left(t, x, L / \varepsilon^{s}\right)$ according to [4, Theorem $3.1 \mathrm{pp}$. 27]. This is the reason why, in the next section, we only study the asymptotic behavior of $p_{p r}\left(t, x, L / \varepsilon^{s}\right)$. Before starting, we also remark that the nature of the source affects strongly the asymptotic shape of the transmitted pulse. Let us investigate the cases of a broadband source $(q<s)$ and a narrowband source $(q=s)$ in the contexts of a homogeneous medium, and then with a random medium in order to understand the effects the random perturbations have on the pulse.

\subsection{Broadband $(q<s)$ and Narrowband $(q=s)$ Pulse in the Homogenous Case}

This section is devoted to the study of the pulse (30) in a homogeneous medium. The results presented in this section will be compared to the ones obtained in Section 3.2 and Section 3.3.

In the homogeneous case we have $\mathbf{P}^{a, \varepsilon}(\omega, L)=I d_{N(\omega)}$, and after the change of variable $\omega=\omega_{0}+\varepsilon^{q} h$ the pulse (30) becomes to leading order

$$
\begin{aligned}
p_{p r}\left(\frac{t}{\varepsilon^{s}}, x, \frac{L}{\varepsilon^{s}}\right) & \underset{\varepsilon \rightarrow 0}{\simeq} \frac{e^{-i \omega_{0} t / \varepsilon^{s}}}{4 \pi} \sum_{j=1}^{N\left(\omega_{0}\right)} \phi_{j}(x)\left\langle\phi_{j}, \Psi\right\rangle_{L^{2}(0, d)} \\
& \times \int e^{-i h\left(t-\beta_{j}^{\prime}\left(\omega_{0}\right) L\right) / \varepsilon^{s-q}} \widehat{f}(h) e^{i \beta_{j}\left(\omega_{0}\right) L / \varepsilon^{s}} e^{i \Phi_{j}^{\varepsilon}(h) L / \varepsilon^{s-2 q}} e^{-i \beta_{j}\left(\omega_{0}\right) L S} d h
\end{aligned}
$$

where

$$
\Phi_{j}^{\varepsilon}(h)=\sum_{n=2}^{n_{q}} \beta_{j}^{(n)}\left(\omega_{0}\right) \varepsilon^{q(n-2)} \frac{h^{n}}{n !},
$$

with $\Phi^{\varepsilon}(h)=0$ if $s<2 q$, and $n_{q}=[s / q]+1$. Here, we made the identification $N\left(\omega_{0}\right)=N\left(\omega_{0}+\varepsilon^{q} h\right)$ since we assumed that $\widehat{f}(h)$ has a compact support and $\varepsilon$ is small. In the broadband case the order of the bandwidth of the source $1 / \varepsilon^{q}$ is small compared to the order of propagation distance $1 / \varepsilon^{s}$, so that a modal dispersion can be observed. In this context, we observe the pulse for a time window of order the pulse width $1 / \varepsilon^{q}$ and centered at $t_{o b s} / \varepsilon^{s}$ which is of order of the total travel from $z=L_{S}$ to $z=L / \varepsilon^{s}$ :

$$
\frac{t}{\varepsilon^{s}}=\frac{t_{o b s}}{\varepsilon^{s}}+\frac{u}{\varepsilon^{q}} \quad \text { with } \quad u \in[-T, T]
$$


Proposition 3.1 For all $j \in\left\{1, \ldots, N\left(\omega_{0}\right)\right\}$, let us consider

$$
p_{j, p r}^{\varepsilon}(u, x, L)=e^{-i \beta_{j}\left(\omega_{0}\right)\left(L / \varepsilon^{s}-L_{S}\right)} e^{i \omega_{0} t_{j} / \varepsilon^{s}} e^{i \omega_{0} u / \varepsilon^{q}} p_{p r}\left(\frac{t_{j}}{\varepsilon^{s}}+\frac{u}{\varepsilon^{q}}, x, \frac{L}{\varepsilon^{s}}\right),
$$

where $s=1 /(2-\mathfrak{h})$ and

$$
t_{j}=\beta_{j}^{\prime}\left(\omega_{0}\right) L
$$

- If $q \in[s / 2, s)$, we have

$$
\lim _{\varepsilon \rightarrow 0} p_{j, p r}^{\varepsilon}(u, x, L)=\frac{1}{2} K_{j, L} * f(u) \phi_{j}(x)\left\langle\phi_{j}, \Psi\right\rangle_{L^{2}(0, d)},
$$

where

$$
\widehat{K}_{j, L}(h)=e^{i h^{2} \beta_{j}^{(2)}\left(\omega_{0}\right) L / 2}, \quad \text { if } \quad q=\frac{s}{2}, \quad \text { and } \quad \widehat{K}_{j, L}(h)=\delta(h), \quad \text { if } \quad q>\frac{s}{2} .
$$

- If $q \in(0, s / 2)$, we have

$$
\lim _{\varepsilon \rightarrow 0} p_{j, p r}^{\varepsilon}(u, x, L)=0
$$

Consequently, in the broadband case, we can observe a train of coherent transmitted pulses at several well separated observation times $t_{j}$. At these times, the pulse is a single mode traveling with the group velocity $1 / \beta_{j}^{\prime}\left(\omega_{0}\right)$ and dispersed through the kernel $K_{j, L}$.

In the narrowband case, the orders of the bandwidth of the source and the propagation distance are the same $\left(\sim 1 / \varepsilon^{s}\right)$ so that the propagating modes overlap, there is no modal dispersion. Consequently, to describe the asymptotic behavior of the pulse we need to compensate the rapid phase $e^{i \beta_{j}(\omega) L / \varepsilon^{s}}$. However, note that (30) is a superposition of $N\left(\omega_{0}\right)$ eigenvector $\phi_{j}$, so that one can study the finite-dimensional vectors corresponding to the modal decomposition compensated by the fast phase as described in the following result.

Proposition 3.2 For all $j \in\left\{1, \ldots, N\left(\omega_{0}\right)\right\}$, let us consider the projection

$$
p_{j, p r}^{\varepsilon}(t, L)=e^{-i \beta_{j}\left(\omega_{0}\right)\left(L / \varepsilon^{s}-L_{S}\right)} e^{i \omega_{0} t / \varepsilon^{s}}\left\langle p_{p r}\left(\frac{t}{\varepsilon^{s}}, \cdot, \frac{L}{\varepsilon^{s}}\right), \phi_{j}\right\rangle_{L^{2}(0, d)} .
$$

We have for $s=1 /(2-\mathfrak{h})$

$$
\lim _{\varepsilon \rightarrow 0} p_{j, p r}^{\varepsilon}(t, L)=\frac{1}{2} f\left(t-\beta_{j}^{\prime}\left(\omega_{0}\right) L\right)\left\langle\phi_{j}, \Psi\right\rangle_{L^{2}(0, d)} .
$$

Roughly speaking, we can then write

$$
p_{p r}\left(\frac{t}{\varepsilon^{s}}, x, \frac{L}{\varepsilon^{s}}\right) \underset{\varepsilon \rightarrow 0}{\simeq} \frac{e^{-i \omega_{0} t / \varepsilon^{s}}}{2} \sum_{j=1}^{N\left(\omega_{0}\right)} f\left(t-\beta_{j}^{\prime}\left(\omega_{0}\right) L\right) e^{i \beta_{j}\left(\omega_{0}\right)\left(L / \varepsilon^{s}-L_{S}\right)} \phi_{j}(x)\left\langle\phi_{j}, \Psi\right\rangle_{L^{2}(0, d)} .
$$

The transmitted pulse is therefore a superposition of modes and each of them is centered around its travel time $t_{j}$ defined by $(32)$.

Let us briefly comment on how we can enhance the source information by forming correlations. Define for any $t_{o b s}>0$ and $j \in\left\{1, \ldots, N\left(\omega_{0}\right)\right\}$

$$
F_{j}^{\varepsilon}(u)=e^{i \omega_{0} u / \varepsilon^{q}} \int\left\langle\phi_{j}, p_{p r}\left(\frac{t_{o b s}}{\varepsilon^{s}}+\frac{u+\tau}{\varepsilon^{q}}, \cdot, L / \varepsilon^{s}\right)\right\rangle_{L^{2}(0, d)}\left\langle\phi_{j}, p_{p r}\left(\frac{t_{o b s}}{\varepsilon^{s}}+\frac{\tau}{\varepsilon^{q}}, \cdot, L / \varepsilon^{s}\right)\right\rangle_{L^{2}(0, d)} d \tau .
$$

Then, we have in the narrow and broad band cases for any $q$ and $t_{o b s}>0$ :

$$
F_{j}^{\varepsilon}(u)=\frac{f^{2 *}(u)}{4}\left\langle\phi_{j}, \Psi\right\rangle_{L^{2}(0, d)}^{2}, \quad \text { for } \quad f^{2 *}(u)=\int f(u+t) f(t) d t .
$$

In the two following sections we are interested in how the pulse is affected by the random perturbations of the propagation medium. We consider first the case of a pulse generated by a broadband source and second by a narrowband source. 


\subsection{Broadband Pulse $(q<s=1 /(2-\mathfrak{h}))$ in the Random Case}

Following the lines of Section 3.1, making the change of variable $\omega=\omega_{0}+\varepsilon^{q} h$ in (30) we have

$$
\begin{aligned}
p_{p r}\left(\frac{t_{o b s}}{\varepsilon^{s}}+\frac{u}{\varepsilon^{q}}, x, \frac{L}{\varepsilon^{s}}\right)=\frac{e^{-i \omega_{0} t_{o b s} / \varepsilon^{s}} e^{-i \omega_{0} u / \varepsilon^{q}}}{4 \pi} & \sum_{j, l=1}^{N\left(\omega_{0}\right)} \sqrt{\frac{\beta_{l}\left(\omega_{0}\right)}{\beta_{j}\left(\omega_{0}\right)}} e^{\beta_{j}\left(\omega_{0}\right) L / \varepsilon^{s}} e^{-i \beta_{l}\left(\omega_{0}\right) L_{S}} \phi_{j}(x)\left\langle\phi_{l}, \Psi\right\rangle_{L^{2}(0, d)} \\
& \times \int e^{-i h u} \widehat{f}(h) e^{i h\left(\beta_{j}^{\prime}\left(\omega_{0}\right) L-t_{o b s}\right) / \varepsilon^{s-q}} e^{i \Phi_{j}^{\varepsilon}(h) L / \varepsilon^{s-2 q}} \\
& \times \mathbf{P}_{j l}^{a, \varepsilon}\left(\omega_{0}+\varepsilon^{q} h, L\right) d h,
\end{aligned}
$$

where $\Phi^{\varepsilon}$ is given by (31). As in the homogeneous case, we can observe a train coherent transmitted pulses, described in the following result, at several well separated observation times $t_{o b s}=t_{j}$ defined by $(32)$.

Theorem 3.1 For all $j \in\left\{1, \ldots, N\left(\omega_{0}\right)\right\}$, let us consider

$$
p_{j, p r}^{\varepsilon}(u, x, L)=e^{-i \beta_{j}\left(\omega_{0}\right)\left(L / \varepsilon^{s}-L_{S}\right)} e^{i \omega_{0} t_{j} / \varepsilon^{s}} e^{i \omega_{0} u / \varepsilon^{q}} p_{p r}\left(\frac{t_{j}}{\varepsilon^{s}}+\frac{u}{\varepsilon^{q}}, x, \frac{L}{\varepsilon^{s}}\right),
$$

where $s=1 /(2-\mathfrak{h})$.

- If $q \in[s / 2, s)$, the family $\left(p_{1, p r}^{\varepsilon}(\cdot, \cdot, L), \ldots, p_{N\left(\omega_{0}\right), p r}^{\varepsilon}(\cdot, \cdot, L)\right)_{\varepsilon}$ converges in distribution on $\mathcal{C}([-T, T] \times$ $\left.(0, d), \mathbb{C}^{N\left(\omega_{0}\right)}\right)$ as $\varepsilon$ goes to 0 to $\left(p_{1, p r}^{0}(\cdot, \cdot, L), \ldots, p_{N\left(\omega_{0}\right), p r}^{0}(\cdot, \cdot, L)\right)_{\varepsilon}$, where

$$
p_{j, p r}^{0}(u, x, L)=\frac{e^{i \sigma_{j, H}\left(\omega_{0}\right) B_{H}(L)}}{2} K_{j, L} * f(u) \phi_{j}(x)\left\langle\phi_{j}, \Psi\right\rangle_{L^{2}(0, d)} .
$$

Here $K_{j, L}$ is defined by (33), $B_{H}$ is a standard fractional Brownian motion with Hurst index $H=(2-\mathfrak{h}) / 2 \in(1 / 2,1)$, and $\sigma_{j, H}\left(\omega_{0}\right)$ is defined by $(28)$.

- If $q \in(0, s / 2)$, the family $\left(p_{1, p r}^{\varepsilon}(\cdot, \cdot, L), \ldots, p_{N\left(\omega_{0}\right), p r}^{\varepsilon}(\cdot, \cdot, L)\right)_{\varepsilon}$ converges in probability on $\mathcal{C}([-T, T] \times$ $\left.(0, d), \mathbb{C}^{N\left(\omega_{0}\right)}\right)$ to 0 as $\varepsilon$ goes to 0.

This result shows that the random perturbations of the propagation medium induce on the pulse a mode-dependent and frequency-dependent phase modulation driven by a single standard fractional Brownian motion, and spread dispersively through the kernel $K_{j, L}$. This result is in contrast with the case of random perturbations with mixing properties (see [9, Section 20.4.3]) and $s=1$ in three respects. First, the random modulation is driven by a fractional Brownian motion and not by a standard Brownian motion. Second, in our case the fractional Brownian motion is the same for all the train of transmitted waves and not a family of standard Brownian motion with some frequency-dependent correlation matrix. Finally, as already discussed in Section 2, on this propagation scale $\left(L / \varepsilon^{s}\right.$ with $\left.s=1 /(2 H)\right)$ the pulse does not accumulate enough scattering events to affect its energy $[11,15]$.

\subsection{Narrowband Pulse $(q=s=1 /(2-\mathfrak{h}))$ in the Random Case}

As discussed in Section 3.1, in the context of a narrowband pulse the propagating modes overlap, there is no modal dispersion, and we have from (30)

$$
\begin{aligned}
p_{p r}\left(\frac{t}{\varepsilon^{s}}, x, \frac{L}{\varepsilon^{s}}\right)=\frac{e^{-i \omega_{0} t / \varepsilon^{s}}}{4 \pi} & \sum_{j, l=1}^{N\left(\omega_{0}\right)} \sqrt{\frac{\beta_{l}\left(\omega_{0}\right)}{\beta_{j}\left(\omega_{0}\right)}} e^{\beta_{j}\left(\omega_{0}\right) L / \varepsilon^{s}} e^{-i \beta_{l}\left(\omega_{0}\right) L_{S}} \phi_{j}(x)\left\langle\phi_{l}, \Psi\right\rangle_{L^{2}(0, d)} \\
& \times \int \widehat{f}(h) e^{i h\left(\beta_{j}^{\prime}\left(\omega_{0}\right) L-t\right)} \\
& \times \mathbf{P}_{j l}^{a, \varepsilon}\left(\omega_{0}+\varepsilon^{s} h, L\right) d h .
\end{aligned}
$$

As in the homogeneous case, because of the mode overlapping we study the finite-dimensional vectors corresponding to the modal decomposition and compensated by the fast phase. The precise result is given in the following theorem. 
Theorem 3.2 For all $j \in\left\{1, \ldots, N\left(\omega_{0}\right)\right\}$, let us consider the projection

$$
p_{j, p r}^{\varepsilon}(t, L)=e^{-i \beta_{j}\left(\omega_{0}\right)\left(L / \varepsilon^{s}-L_{S}\right)} e^{i \omega_{0} t / \varepsilon^{s}}\left\langle p_{p r}\left(\frac{t}{\varepsilon^{s}}, \cdot \frac{L}{\varepsilon^{s}}\right), \phi_{j}\right\rangle_{L^{2}(0, d)} .
$$

The family $\left(p_{1, p r}^{\varepsilon}(\cdot, L), \ldots, p_{N\left(\omega_{0}\right), p r}^{\varepsilon}(\cdot, L)\right)_{\varepsilon}$ converges in distribution in $\mathcal{C}\left([-T, T], \mathbb{C}^{N\left(\omega_{0}\right)}\right)$ as $\varepsilon$ goes to 0 to $\left(p_{1, p r}^{0}(\cdot, L), \ldots, p_{N\left(\omega_{0}\right), p r}^{0}(\cdot, L)\right)_{\varepsilon}$, where for $j \in\left\{1, \ldots, N\left(\omega_{0}\right)\right\}$

$$
p_{j, p r}^{0}(t, L)=\frac{1}{2} e^{i \sigma_{j, H}\left(\omega_{0}\right) B_{H}(L)} f\left(t-\beta_{j}^{\prime}\left(\omega_{0}\right) L\right)\left\langle\phi_{j}, \Psi\right\rangle_{L^{2}(0, d)} .
$$

Here, $B_{H}$ is a standard fractional Brownian motion with Hurst index $H \in(1 / 2,1)$, and $\sigma_{j, H}\left(\omega_{0}\right)$ is defined by (28).

Roughly speaking, the pulse can be described as

$$
p_{p r}\left(\frac{t}{\varepsilon^{s}}, x, \frac{L}{\varepsilon^{s}}\right) \underset{\varepsilon \rightarrow 0}{\simeq} \frac{e^{-i \omega_{0} t / \varepsilon^{s}}}{2} \sum_{j=1}^{N\left(\omega_{0}\right)} e^{i \sigma_{j, H}\left(\omega_{0}\right) B_{H}(L)} f\left(t-\beta_{j}^{\prime}\left(\omega_{0}\right) L\right) e^{i \beta_{j}\left(\omega_{0}\right)\left(L / \varepsilon^{s}-L_{S}\right)} \phi_{j}(x)\left\langle\phi_{j}, \Psi\right\rangle_{L^{2}(0, d)} .
$$

The transmitted pulse is therefore a superposition of modes, each of them is centered around its travel time $t_{j}$ defined by (32), but also modulated by a mode-dependent and frequency-dependent random phase. Once again the randomness comes from the same fractional Brownian motion for all the propagating modes.

In the way as in the homogeneous case, one can enhance the source information by forming correlations. Considering $F_{j}^{\varepsilon}(u)$ given by (35), we have according to Theorem 2.2 , both in the narrow and broad band cases, for any $q$ and $t_{o b s}>0$

$$
\lim _{\varepsilon \rightarrow 0} F_{j}^{\varepsilon}(u)=\frac{f^{2 *}(u)}{4}\left\langle\phi_{j}, \Psi\right\rangle_{L^{2}(0, d)}^{2},
$$

with $f^{2 *}$ given by (36), and where the limit holds in probability. Here, we recover the same result has in the homogeneous case. This is due to the diagonal form of the limit process in Theorem 2.2 and the correlations which compensate the phases.

\section{Pulse Propagation for a Single-Mode Waveguide with Neu- mann Boundary Conditions}

In this section we study the particular case of a single-mode waveguide with Neumann boundary conditions on $\partial \mathcal{D}$ to make the link with earlier works in one-dimensional propagation media [17, 18]. In this context, the spectral analysis of the transverse Laplacian $\partial_{x}^{2}$ in (8) is a bit different : $-\partial_{x}^{2}$ with Neumann boundary conditions on $\partial \mathcal{D}$ is a nonnegative self-adjoint operator in $L^{2}(0, d)$. Its spectrum is constituted of a countably infinite number of nonnegative eigenvalues $\left(\lambda_{j}\right)_{j \geq 0}$ since $(0, d)$ is a bounded domain. Therefore, we have for all $j \geq 0$

$$
-\partial_{x}^{2} \phi_{j}(x)=\lambda_{j} \phi_{j}(x) \quad \forall x \in(0, d), \quad \text { and } \quad \phi_{j}^{\prime}(0)=\phi_{j}^{\prime}(d)=0,
$$

where $0=\lambda_{0}<\lambda_{1}<\cdots$ and the eigenvectors $\left(\phi_{j}\right)_{j \geq 0}$ form an orthonormal basis of $L^{2}(0, d)$. In our context, we have explicit expressions for the eigenvectors and eigenvalues

$$
\lambda_{0}=0 \quad \text { and } \quad \phi_{0}=\frac{1}{\sqrt{d}},
$$

and

$$
\lambda_{j}=\frac{j^{2} \pi^{2}}{d^{2}} \quad \text { and } \quad \phi_{j}(x)=\sqrt{\frac{2}{d}} \cos (j \pi x / d), \quad \text { for } j \geq 1
$$

Moreover, in this context the number of propagating modes is now given by

$$
N(\omega)=1+\left[\frac{\omega d}{\pi c}\right]
$$


so that in order to have only one propagating mode $(N(\omega)=1)$ on need to take

$$
\omega \in\left(-\omega_{c}, \omega_{c}\right) \quad \text { with } \quad \omega_{c}=c \sqrt{\lambda_{1}}
$$

Let us assume that the temporal profile of the source (2) is given by $f^{\varepsilon}(t)=f(t)$, for which the support of $\widehat{f}(\omega)$ is included in $\left(-\omega_{c}, \omega_{c}\right)$. According to the remarks at the beginning of Section 3 , to study the asymptotic behavior of the pulse, we consider the following propagating part with only one mode:

$$
p_{p r}\left(t, \frac{L}{\varepsilon^{s}}\right)=\frac{\int_{0}^{d} \Psi(x) d x}{4 \pi d} \int \widehat{f}(\omega) e^{-i \omega\left(t-L /\left(c \varepsilon^{s}\right)\right)}\left[\overline{\mathbf{P}}_{11}^{a, \varepsilon}(\omega, L)\right]^{-1} d \omega
$$

Consequently, we observe the transmitted pulse in a time window of order 1 (comparable to the pulse width) and centered at $L /\left(c \varepsilon^{s}\right)$ (of order the total travel time)

$$
p_{p r}^{\varepsilon}(u, L)=p_{p r}\left(\frac{L}{c \varepsilon^{s}}+u, \frac{L}{\varepsilon^{s}}\right)=\frac{\int_{0}^{d} \Psi(x) d x}{4 \pi d} \int \widehat{f}(\omega) e^{-i \omega u}\left[\overline{\mathbf{P}_{11}^{a, \varepsilon}}(\omega, L)\right]^{-1} d \omega,
$$

and we have the following result.

Theorem 4.1 For $s=1 /(2-\mathfrak{h})$ the family $\left(p_{p r}^{\varepsilon}(\cdot, L)\right)_{\varepsilon}$ converges in distribution on $\mathcal{C}([-T, T])$ as $\varepsilon$ goes to 0 to

$$
p_{p r}^{0}(t, L)=\frac{\int_{0}^{d} \Psi(x) d x}{2 d} f\left(t-\sigma_{H} B_{H}(L)\right) .
$$

Here, $B_{H}$ is a standard fractional Brownian motion with Hurst index $H=(2-\mathfrak{h}) / 2 \in(1 / 2,1)$, and

$$
\sigma_{H}^{2}=\frac{C_{\mathfrak{h}}}{4 H(2 H-1) d^{2} c^{2}} \int_{(0, d)^{2}} R\left(x, x^{\prime}\right) d x d x^{\prime} .
$$

The proof of this result follows closely the one of Theorem 3.1 and Theorem 3.2 using the first point of Theorem 2.2. The result obtained in Theorem 4.1 is similar to the ones obtained in [17, 18] for onedimensional propagation media. The transmitted pulse is the original pulse with a random time shift given by a fractional Brownian motion. In contrast with the result obtained in the same context (singlemode random waveguide) but with random perturbations having mixing properties [10, Proposition 3] the random shift is a fractional Brownian motion and not a standard Brownian motion, and there is no determinist deformation of the pulse. Moreover, the energy is not affected because the pulse does not accumulate enough scattering events to affect it.

In the remaining part of the paper we derive some properties on the stochastic process $V(z, x)$ which we then use in the proof of Theorem 2.1, Theorem 3.1, and Theorem 3.2.

\section{The random fluctuations model}

This section is devoted to some properties of the random field $V(z, x)$ (defined by (3)) modeling the medium fluctuations. The long-range property (4) is the key to observe stochastic effects driven by a fractional Brownian motion and not a standard one as in the mixing case [9, Chapter 20]. The following proposition shows that $V$ exhibits the long-range property as well.

Proposition 5.1 For all $s \in \mathbb{R}$ and $(x, y) \in(0, d)^{2}$, we have

$$
\mathbb{E}[V(z+s, x) V(s, y)] \underset{z \rightarrow+\infty}{\sim} \frac{C_{\mathfrak{h}}}{z^{\mathfrak{h}}} R(x, y) \quad \text { with } \quad C_{\mathfrak{h}}=\frac{c_{\mathfrak{h}}}{2 \pi}\left(\int_{-\infty}^{+\infty} \Theta(x) x e^{-x^{2} / 2} d x\right)^{2} .
$$

The proof of this proposition follows exactly the line of [17, Lemma 1], but we present it in this section as preliminaries for the proof of Proposition 5.2.

Proof (of Proposition 5.1) Let us first remind the reader that the Hermite polynomials (6) form an orthogonal basis of $L^{2}(\mathbb{R}, w(x) d x)$ such that

$$
\left\langle H_{l}, H_{m}\right\rangle_{L^{2}(\mathbb{R}, w(x) d x)}=l ! \delta_{l m},
$$


and let us recall the Mehler's formula, which is

$$
\mathbb{E}\left[H_{l}\left(X_{1}\right) H_{m}\left(X_{2}\right)\right]=l ! \mathbb{E}\left[X_{1} X_{2}\right]^{l} \delta_{l m}
$$

for two centered Gaussian random variables such that $\mathbb{E}\left[X_{1}^{2}\right]=\mathbb{E}\left[X_{2}^{2}\right]=1$. Decomposing $\Theta$ in this basis

$$
\Theta(x)=\sum_{l \geq 1} \frac{\Theta_{l}}{l !} H_{l}(x), \quad \text { where } \quad \Theta_{l}=\left\langle H_{l}, \Theta\right\rangle_{L^{2}(\mathbb{R}, w(x) d x)},
$$

and using the Mehler formula we have

$$
\begin{aligned}
\mathbb{E}\left[\Theta\left(\mathcal{B}_{\mathfrak{h}}(z+s, x)\right) \Theta\left(\mathcal{B}_{\mathfrak{h}}(s, y)\right)\right] & =\sum_{l, m \geq 1} \frac{\Theta_{l} \Theta_{m}}{l ! m !} \mathbb{E}\left[H_{l}\left(\mathcal{B}_{\mathfrak{h}}(z+s, x)\right) H_{m}\left(\mathcal{B}_{\mathfrak{h}}(s, y)\right)\right] \\
& =\sum_{l \geq 1} \frac{\Theta_{l}^{2}}{l !} r_{\mathfrak{h}}^{l}(z) R^{l}(x, y) \\
& =\Theta_{1}^{2} r_{\mathfrak{h}}(z) R(x, y)+\sum_{l \geq 2} \frac{\Theta_{l}^{2}}{l !} r_{\mathfrak{h}}^{l}(z) R^{l}(x, y) .
\end{aligned}
$$

Now, let us remark that for $l \geq 2$ we have $z^{\mathfrak{h}} r_{\mathfrak{h}}^{l}(z) \rightarrow 0$ as $z \rightarrow+\infty$, and for $z$ large enough

$$
\sum_{l \geq 2}\left|\frac{\Theta_{l}^{2}}{l !} r_{\mathfrak{h}}^{l}(z) R^{l}(x, y)\right| \leq C \sum_{l \geq 2} \frac{\Theta_{l}^{2}}{l !}<+\infty,
$$

since for all $l \geq 1$ we have assumed (5). Therefore, we finally have

$$
z^{\mathfrak{h}} \mathbb{E}[V(z+s, x) V(s, y)] \underset{z \rightarrow+\infty}{\sim} c_{\mathfrak{h}} \Theta_{1}^{2} R(x, y),
$$

which concludes the proof of Proposition 5.1.

The proof of Theorem 2.1 is based on a moment techniques, so that we have to evaluate moments of the form

$$
\frac{1}{\varepsilon^{n(s-1 / 2)}} \int_{\Delta_{n}(z)} \mathbb{E}\left[\prod_{p=1}^{n} \Theta\left(\mathcal{B}_{\mathfrak{h}}\left(\frac{z_{p}}{\varepsilon^{s}}, x_{p}\right)\right)\right] \varphi_{\varepsilon}\left(z, z_{1}, \ldots, z_{n}\right) d z_{1} \ldots d z_{n}
$$

where $n$ is an even number (otherwise this moment is 0 by symmetry), and

$$
\Delta_{n}(z)=\left\{\left(z_{1}, \ldots, z_{n}\right) \in[0, z]^{n}, \quad \text { s.t. } \quad 0 \leq z_{j} \leq z_{j-1} \quad \forall j \in\{2, \ldots, n\}\right\} .
$$

The following proposition gives the leading order term of these moments.

Proposition 5.2 For all even number $n \geq 2,\left(x_{1}, \ldots, x_{n}\right) \in(0, d)^{n}$ and $s=1 /(2-\mathfrak{h})$, there exists a positive constant $C$ such that

$$
\sup _{\varepsilon \in(0,1)} \frac{1}{\varepsilon^{n(s-1 / 2)}} \int_{[0, z]^{n}}\left|\mathbb{E}\left[\prod_{p=1}^{n} \Theta\left(\mathcal{B}_{\mathfrak{h}}\left(\frac{z_{p}}{\varepsilon^{s}}, x_{p}\right)\right)\right]\right| d z_{1} \ldots d z_{n} \leq C^{n},
$$

and

$$
\begin{aligned}
\lim _{\varepsilon \rightarrow 0} \frac{1}{\varepsilon^{n(s-1 / 2)}} \int_{\Delta_{n}(z)} \mathbb{E}\left[\prod_{p=1}^{n} \Theta\left(\mathcal{B}_{\mathfrak{h}}\left(\frac{z_{p}}{\varepsilon^{s}}, x_{p}\right)\right)\right] \varphi_{\varepsilon}\left(z, z_{1}, \ldots, z_{n}\right) d z_{1} \ldots d z_{n} \\
\quad=\lim _{\varepsilon \rightarrow 0} C_{\mathfrak{h}}^{n / 2} \int_{\Delta_{n}(z)} \sum_{\mathcal{F}} \prod_{(p, q) \in \mathcal{F}} \frac{R\left(x_{p}, x_{q}\right)}{\left|z_{p}-z_{q}\right|^{\mathfrak{h}}} \varphi_{\varepsilon}\left(z, z_{1}, \ldots, z_{n}\right) d z_{1} \ldots d z_{n}
\end{aligned}
$$

where $\varphi_{\varepsilon}$ is a bounded function for all $\varepsilon$. Here, the sum stands over the parings $\mathcal{F}$ of $\{1, \ldots, n\}$, and the limit $\varepsilon \rightarrow 0$ is uniform with respect to $\left(x_{1}, \ldots, x_{n}\right)$.

We recall that a pairing formed over vertices of $S=\{1, \ldots, n\}$ is a partition of $S$ into $n$ pairs of couple $(p, q)$ such that all the elements of $S$ appear only in one of the pairs and with $p<q$. 
Proof (of Proposition 5.2) The proof of this result is inspired from [22]. For the first point, using the decomposition of the function $x \mapsto \Theta(\sqrt{2 n} x)$ over the orthogonal basis of Hermite polynomials we have

$$
\begin{aligned}
\mathbb{E}\left[\prod_{p=1}^{n} \Theta\left(\mathcal{B}_{\mathfrak{h}}\left(\frac{z_{p}}{\varepsilon^{s}}, x_{p}\right)\right)\right] & =\mathbb{E}\left[\prod_{p=1}^{n} \Theta\left(\sqrt{2 n} \frac{1}{\sqrt{2 n}} \mathcal{B}_{\mathfrak{h}}\left(\frac{z_{p}}{\varepsilon^{s}}, x_{p}\right)\right)\right] \\
& =\sum_{\substack{l_{j} \geq 1 \\
j \in\{1, \ldots, n\}}} \prod_{p=1}^{n} \frac{\Theta_{n, l_{p}}}{l_{p} !} \mathbb{E}\left[\prod_{p=1}^{n} H_{l_{p}}\left(\frac{1}{\sqrt{2 n}} \mathcal{B}_{\mathfrak{h}}\left(\frac{z_{p}}{\varepsilon^{s}}, x_{p}\right)\right)\right] .
\end{aligned}
$$

Then, following the proof of [22, Lemma 4.5] and using [22, Corollary 4.2] we have

$$
\begin{aligned}
\int_{[0, z]^{n}} \mid \mathbb{E} & {\left[\prod_{p=1}^{n} \Theta\left(\mathcal{B}_{\mathfrak{h}}\left(\frac{z_{p}}{\varepsilon^{s}}, x_{p}\right)\right)\right] \mid d z_{1} \ldots d z_{n} \leq 2^{n+2} z^{n / 2}\left(\frac{\|R\|_{\infty}}{2 n} \int_{0}^{z} r_{\mathfrak{h}}\left(\frac{u}{\varepsilon^{s}}\right) d u\right)^{n / 2} } \\
& \times \sum_{q \geq n / 2}\left(\frac{1}{2 n}\right)^{q-n / 2} \sum_{\begin{array}{c}
k_{1}+\cdots+k_{n}=2 q \\
k_{1}, \ldots, k_{n} \in\{1, \ldots, q\}
\end{array}} \prod_{p=1}^{n} \frac{\left|\Theta_{n, k_{p}}\right|}{k_{p} !} \mathbb{E}\left[\left|\prod_{p=1}^{n} H_{k_{p}}(X)\right|\right],
\end{aligned}
$$

where $X \sim \mathcal{N}(0,1)$, and for which according to [22, Lemma 3.1] we have

$$
\mathbb{E}\left[\left|\prod_{p=1}^{n} H_{k_{p}}(X)\right|\right] \leq \prod_{p=1}^{n}(n-1)^{k_{p} / 2} \sqrt{k_{p} !} \quad \text { and } \quad\left|\Theta_{n, l}\right| \leq \sup |\Theta| \sqrt{l !} .
$$

Moreover, according to (4), there exists $z_{e}$ such that for all $z>z_{e}$ we have $\left|r_{\mathfrak{h}}(z)\right| \leq C|z|^{-\mathfrak{h}}$, and then

$$
\left|\int_{0}^{z} r_{\mathfrak{h}}\left(\frac{u}{\varepsilon^{s}}\right) d u\right| \leq C\left(\varepsilon^{s}+\varepsilon^{s \mathfrak{h}} \int_{\varepsilon^{s} z_{e}}^{z}|u|^{-\mathfrak{h}} d u\right) .
$$

As a result, for $s=1 /(2-\mathfrak{h})$ we finally obtain

$$
\frac{1}{\varepsilon^{n(s-1 / 2)}} \int_{[0, z]^{n}}\left|\mathbb{E}\left[\prod_{p=1}^{n} \Theta\left(\mathcal{B}_{\mathfrak{h}}\left(\frac{z_{p}}{\varepsilon^{s}}, x_{p}\right)\right)\right]\right| d z_{1} \ldots d z_{n} \leq C^{n}\left(\sum_{l \geq 1}\left(\frac{n-1}{2 n}\right)^{l / 2}\right)^{n}=\tilde{C}^{n} .
$$

To prove the second point, let us decompose $\Theta$ over the orthogonal basis of Hermite polynomials,

$$
\begin{aligned}
\mathbb{E}\left[\prod_{p=1}^{n} \Theta\left(\mathcal{B}_{\mathfrak{h}}\left(\frac{z_{p}}{\varepsilon^{s}}, x_{p}\right)\right)\right] & =\sum_{\substack{l_{j} \geq 1 \\
j \in\{1, \ldots, n\}}} \prod_{p=1}^{n} \frac{\Theta_{l_{p}}}{l_{p} !} \mathbb{E}\left[\prod_{p=1}^{n} H_{l_{p}}\left(\mathcal{B}_{\mathfrak{h}}\left(\frac{z_{p}}{\varepsilon^{s}}, x_{p}\right)\right)\right] \\
& =\Theta_{1}^{n} \sum_{\mathcal{F}} \prod_{(p, q) \in \mathcal{F}} r_{\mathfrak{h}}\left(\frac{z_{p}-z_{q}}{\varepsilon^{s}}\right) R\left(x_{p}, x_{q}\right) \\
& +\mathcal{I}_{n}^{\varepsilon}\left(z_{1}, \ldots, z_{n}, x_{1}, \ldots, x_{n}\right)
\end{aligned}
$$

where

$$
\begin{aligned}
& \mathcal{I}_{n}^{\varepsilon}\left(z_{1}, \ldots, z_{n}, x_{1}, \ldots, x_{n}\right)=\sum_{p_{0}=1}^{n} \sum_{\substack{l_{j} \geq 1 \\
\text { for } p \neq p_{0} \\
\text { and } p_{0} \geq 2}} \prod_{p=1}^{n} \frac{\Theta_{l_{p}}}{l_{p} !} \mathbb{E}\left[\prod_{p=1}^{n} H_{l_{p}}\left(\mathcal{B}_{\mathfrak{h}}\left(\frac{z_{p}}{\varepsilon^{s}}, x_{p}\right)\right)\right] \\
& =\sum_{p_{0}=1}^{n} \sum_{q \geq n / 2+1} \sum_{\substack{l_{1}+\ldots+l_{n}=2 q \\
l_{p} \in\{1, \ldots, q\} \text { for } p \neq p_{0} \\
l_{p_{0}} \in\{2, \ldots, q\}}} \prod_{p=1}^{n} \frac{\Theta_{l_{p}}}{l_{p} !} \mathbb{E}\left[\prod_{p=1}^{n} H_{l_{p}}\left(\mathcal{B}_{\mathfrak{h}}\left(\frac{z_{p}}{\varepsilon^{s}}, x_{p}\right)\right)\right] .
\end{aligned}
$$

Here, let us remark that $q \geq n / 2+1$ since $n$ is even and $l_{p_{0}} \geq 2$. Moreover, according to [22, Lemma 2.1 , we have

$$
\mathbb{E}\left[\prod_{p=1}^{n} H_{l_{p}}\left(\mathcal{B}_{\mathfrak{h}}\left(\frac{z_{p}}{\varepsilon^{s}}, x_{p}\right)\right)\right]=\left\{\begin{array}{l}
\frac{l_{1} ! \ldots . l_{n} !}{2^{q} q !} \sum_{I\left(l_{1}, \ldots, l_{n}\right)} \prod_{p=1}^{q} r_{\mathfrak{h}}\left(\frac{z_{i_{p}}-z_{j_{p}}}{\varepsilon^{s}}\right) \hat{R}\left(x_{i_{p}}, x_{j_{p}}\right) \\
\text { if } l_{1}+\cdots+l_{n}=2 q \\
0 \quad \text { otherwise }
\end{array}\right.
$$


where

$$
\begin{gathered}
I\left(l_{1}, \ldots, l_{n}\right)=\left\{\left(i_{1}, j_{1}, \ldots, i_{q}, j_{q}\right) \in\{1, \ldots, n\}^{2 q}, \quad \text { s.t. } i_{p} \neq j_{p} \quad \forall p \in\{1, \ldots, q\}\right. \\
\text { and all } \left.p \in\{1, \ldots, n\} \quad \text { appears } l_{p} \text { times }\right\} .
\end{gathered}
$$

Using the fact that $r_{\mathfrak{h}}$ and $R$ are assumed to be bounded by 1 , for all $\left(i_{1}, j_{1}, \ldots, i_{q}, j_{q}\right) \in I\left(l_{1}, \ldots, l_{n}\right)$ we have

$$
\int_{[0, z]^{n}} \prod_{p=1}^{q}\left|r_{\mathfrak{h}}\left(\frac{z_{i_{p}}-z_{j_{p}}}{\varepsilon^{s}}\right) R\left(x_{i_{p}}, x_{j_{p}}\right)\right| d z_{1} \ldots d z_{n} \leq \int_{[0, z]^{n}} \prod_{p=1}^{n / 2+1}\left|r_{\mathfrak{h}}\left(\frac{z_{i_{p}^{\prime}}-z_{j_{p}^{\prime}}}{\varepsilon^{s}}\right)\right| d z_{1} \ldots d z_{n},
$$

where $\left(i_{1}^{\prime}, j_{1}^{\prime}, \ldots, i_{n / 2+1}^{\prime}, j_{n / 2+1}^{\prime}\right)$ repeat $p_{0}$ two times. Since $n / 2+1$ is odd, exactly one other index denoted $p_{0}^{\prime}$ appears also two times, and then two cases are possible. The first case is when we have a term of the form $r_{\mathfrak{H}}^{2}$, if it is the case there is only one, and we have

$$
\int_{0}^{z} d z_{p_{0}} \int_{0}^{z} d z_{p_{0}^{\prime}} r_{\mathfrak{h}}^{2}\left(\frac{z_{p_{0}}-z_{p_{0}^{\prime}}}{\varepsilon^{s}}\right) \leq\left\{\begin{array}{cll}
C_{1} \varepsilon^{2 s \mathfrak{h}} & \text { if } & \mathfrak{h} \in(0,1 / 2), \\
C_{1}^{\prime} \varepsilon^{s} \log \left(1 / \varepsilon^{s}\right) & \text { if } & \mathfrak{h}=1 / 2, \\
C_{1}^{\prime \prime} \varepsilon^{s} & \text { if } & \mathfrak{h} \in(1 / 2,1),
\end{array},\right.
$$

Using (41), we finally have

$$
\begin{aligned}
\int_{[0, z]^{n}} \prod_{p=1}^{n / 2+1}\left|r_{\mathfrak{h}}\left(\frac{z_{i_{p}^{\prime}}-z_{j_{p}^{\prime}}}{\varepsilon^{s}}\right)\right| d z_{1} \ldots d z_{n} & =\left(\int_{0}^{z} d u \int_{0}^{z} d v\left|r_{\mathfrak{h}}\left(\frac{u-v}{\varepsilon^{s}}\right)\right| d u d v\right)^{n / 2-1} \\
& \times \int_{0}^{z} d u \int_{0}^{z} d v r_{\mathfrak{h}}^{2}\left(\frac{u-v}{\varepsilon^{s}}\right) d u d v \\
& \leq C_{2} \varepsilon^{s \mathfrak{s h n} / 2} \varepsilon^{s \mathfrak{h} \wedge(1-\mathfrak{h})} \log \left(1 / \varepsilon^{s}\right) .
\end{aligned}
$$

Second, If we are not in the previous case, using the Cauchy-Schwarz's inequality with respect to $z_{p_{0}}$, a change of variable, and again (41), we obtain

$$
\begin{aligned}
\int_{[0, z]^{n}} \prod_{p=1}^{n / 2+1}\left|r_{\mathfrak{h}}\left(\frac{z_{i_{p}^{\prime}}-z_{j_{m}^{\prime}}}{\varepsilon^{s}}\right)\right| d z_{1} \ldots d z_{n} & \leq C_{3}\left(\int_{0}^{z} d u \int_{0}^{z} d v\left|r_{\mathfrak{h}}\left(\frac{u-v}{\varepsilon^{s}}\right)\right| d u d v\right)^{n / 2-1} \\
& \times \int_{0}^{2 z} d u r_{\mathfrak{h}}^{2}\left(\frac{u}{\varepsilon}\right) d u \\
& \leq C_{4} \varepsilon^{s \mathfrak{h} n / 2} \varepsilon^{s \mathfrak{h} \wedge(1-\mathfrak{h})} \log \left(1 / \varepsilon^{s}\right)
\end{aligned}
$$

Therefore, in any case we have

$$
\begin{aligned}
\int_{[0, z]^{n}} \sup _{x_{1}, \ldots, x_{n}}\left|\mathbb{E}\left[\prod_{p=1}^{n} H_{l_{p}}\left(\mathcal{B}_{\mathfrak{h}}\left(\frac{z_{p}}{\varepsilon^{s}}, x_{p}\right)\right)\right]\right| d z_{1} \ldots d z_{n} \\
\quad \leq C_{4} \varepsilon^{s \mathfrak{s h n} / 2} \varepsilon^{s \mathfrak{h} \wedge(1-\mathfrak{h})} \log \left(1 / \varepsilon^{s}\right)\left|\mathbb{E}\left[\prod_{p=1}^{n} H_{l_{p}}(X)\right]\right|
\end{aligned}
$$

using [22, Lemma 3.2] with $X \sim \mathcal{N}(0,1)$. Consequently, we obtain

$$
\begin{aligned}
& \frac{1}{\varepsilon^{n(s-1 / 2)}} \int_{[0, z]^{n}}\left|\mathcal{I}_{n}^{\varepsilon}\left(z_{1}, \ldots, z_{n}, x_{1}, \ldots, x_{n}\right)\right| d z_{1} \ldots d z_{n} \\
& \leq \varepsilon^{s \mathfrak{h} \wedge(1-\mathfrak{h})} \log \left(1 / \varepsilon^{s}\right) n \sum_{q \geq 1} \sum_{\substack{l_{1}+\ldots+l_{n}=2 q \\
1 \leq l_{p} \leq q}} \prod_{p=1}^{n} \frac{\left|\Theta_{l_{p}}\right|}{l_{p} !} \mathbb{E}\left[\prod_{p=1}^{n}\left|H_{l_{p}}(X)\right|\right],
\end{aligned}
$$

where $X \sim \mathcal{N}(0,1)$, and

$$
\mathbb{E}\left[\prod_{p=1}^{n}\left|H_{l_{p}}(X)\right|\right] \leq(n-1)^{\left(l_{1}+\cdots+l_{n}\right) / 2} \prod_{p=1}^{n} \sqrt{l_{p} !}
$$


using [22, Lemma 3.1]. As a result, we have

$$
\begin{gathered}
\frac{1}{\varepsilon^{n(s-1 / 2)}} \int_{\Delta_{n}(z)}\left|\mathbb{E}\left[\prod_{p=1}^{n} \Theta\left(\mathcal{B}_{\mathfrak{h}}\left(\frac{z_{p}}{\varepsilon^{s}}, x_{p}\right)\right)\right]-\Theta_{1}^{n} \sum_{\mathcal{F}} \prod_{(p, q) \in \mathcal{F}} r_{\mathfrak{h}}\left(\frac{z_{p}-z_{q}}{\varepsilon^{s}}\right) R\left(x_{p}, x_{q}\right)\right| d z_{1} \ldots d z_{n} \\
\leq \varepsilon^{s \mathfrak{h} \wedge(1-\mathfrak{h})} \log \left(1 / \varepsilon^{s}\right) \frac{C^{n}}{(n-1) !}\left(\sum_{l \geq 1} \frac{\left|\Theta_{l}\right|(n-1)^{l / 2}}{\sqrt{l !}}\right)^{n} \\
\leq \varepsilon^{\mathfrak{h} /(2-\mathfrak{h})} \frac{\tilde{C}_{n}^{n}}{(n-1) !}
\end{gathered}
$$

since $s=1 /(2-\mathfrak{h})$ and according to (5). Finally, we have

$$
\begin{aligned}
\sum_{\mathcal{F}} \frac{1}{\varepsilon^{n(s-1 / 2)}} \int_{\Delta_{n}(z)} \prod_{(p, q) \in \mathcal{F}} \mid & r_{\mathfrak{h}}\left(\frac{z_{p}-z_{q}}{\varepsilon^{s}}\right)-\frac{\varepsilon^{s \mathfrak{h}} c_{\mathfrak{h}}}{\left|z_{p}-z_{q}\right|^{\mathfrak{h}}} \mid d z_{1} \ldots d z_{n} \\
\leq & \frac{(n-1) ! !}{n !}\left[\frac{1}{\varepsilon^{(2 s-1)}} \int_{0}^{z} \int_{0}^{z}\left|r_{\mathfrak{h}}\left(\frac{u-v}{\varepsilon^{s}}\right)-\frac{\varepsilon^{s \mathfrak{h}} c_{\mathfrak{h}}}{|u-v|^{\mathfrak{h}}}\right| d u d v\right]^{n / 2}
\end{aligned}
$$

where $(n-1) ! !=n ! /\left(2^{n / 2}(n / 2) !\right)$ is the number of pairing of $\{1, \ldots, n\}$. Moreover, according to (4), for all $\delta>0$, there exists $z_{e}$ such that for all $z>z_{e}$ we have $\left.\left.\left|r_{\mathfrak{h}}(z)-c_{\mathfrak{h}}\right| z\right|^{-\mathfrak{h}}\left|\leq \delta c_{\mathfrak{h}}\right| z\right|^{-\mathfrak{h}}$. Therefore, we have

$$
\begin{aligned}
& \frac{1}{\varepsilon^{(2 s-1)}} \int_{0}^{z} \int_{0}^{z}\left|r_{\mathfrak{h}}\left(\frac{u-v}{\varepsilon^{s}}\right)-\frac{\varepsilon^{s \mathfrak{h}} c_{\mathfrak{h}}}{|u-v|^{\mathfrak{h}}}\right| d u d v \leq \delta c_{\mathfrak{h}} \int_{|u-v|>\varepsilon^{s} z_{e}}|u-v|^{-\mathfrak{h}} d u d v \\
& +\varepsilon \int_{|u-v| \leq z_{a}} r_{\mathfrak{h}}(u-v) d u d v \\
& +c_{\mathfrak{h}} \int_{|u-v| \leq \varepsilon^{s} z_{a}}|u-v|^{-\mathfrak{h}} d u d v
\end{aligned}
$$

so that for all $\delta>0$

$$
\varlimsup_{\varepsilon \rightarrow 0} \frac{1}{\varepsilon^{(2 s-1)}} \int_{0}^{z} \int_{0}^{z}\left|r_{\mathfrak{h}}\left(\frac{u-v}{\varepsilon^{s}}\right)-\frac{\varepsilon^{s \mathfrak{h}} c_{\mathfrak{h}}}{|u-v|^{\mathfrak{h}}}\right| d u d v \leq \delta c_{\mathfrak{h}} \int_{0}^{z} \int_{0}^{z}|u-v|^{-\mathfrak{h}} d u d v,
$$

which concludes the proof of the Proposition 5.2.

\section{Proof of Theorem 2.1}

The proof of this theorem is based on the idea developed in [3] in which the authors study the asymptotic behavior of the solution of the random Schrödinger equation with long-range correlations. The technique is based on the characterization of the moments of the limiting process. This technique is very convenient in our case. In fact, even if the field $V(z, x)$ in $(3)$ is not Gaussian, the field $\mathcal{B}_{\mathfrak{h}}$ is Gaussian and the moments of $V$ can be managed as described in Proposition 5.2.

To apply the moment technique to $\mathcal{P}^{\varepsilon}(\omega, z)$, we perform iterations of the integrated form of $(25)$,

$$
\mathcal{P}^{\varepsilon}(\omega, z)=\left[\begin{array}{l}
\mathbf{P}^{a, \varepsilon}(\omega, z) \\
\mathbf{P}^{b, \varepsilon}(\omega, z)
\end{array}\right]=\left[\begin{array}{c}
I d_{N} \\
\mathbf{0}
\end{array}\right]+\int_{0}^{z}\left[\frac{1}{\varepsilon^{s-1 / 2}} \mathbf{H}\left(\omega, \frac{u}{\varepsilon^{s}}\right)+\varepsilon^{1-s} \mathbf{G}\left(\omega, \frac{u}{\varepsilon^{s}}\right)\right] \mathcal{P}^{\varepsilon}(\omega, u) d u,
$$

so that the transfer operator is given in term of a series

$$
\mathcal{P}^{\varepsilon}(\omega, z)=\sum_{n=0}^{+\infty} \mathcal{P}^{\varepsilon, n}(\omega, z)
$$

where

$$
\mathcal{P}^{\varepsilon, n}(\omega, z)=\int \cdots \int_{\Delta_{n}(z)} \prod_{m=1}^{n} d u_{m} \prod_{m=1}^{n}\left[\frac{1}{\varepsilon^{s-1 / 2}} \mathbf{F}^{1, \varepsilon}\left(\omega, u_{m}\right)+\varepsilon^{1-s} \mathbf{F}^{2, \varepsilon}\left(\omega, u_{m}\right)\right]\left[\begin{array}{c}
I d_{N} \\
\mathbf{0}
\end{array}\right]
$$


and

$$
\Delta_{n}(z)=\left\{\left(z_{1}, \ldots, z_{n}\right) \in[0, z]^{n}, \quad \text { s.t. } \quad 0 \leq z_{j} \leq z_{j-1} \quad \forall j \in\{2, \ldots, n\}\right\} .
$$

Here, we use the notation

$$
\begin{array}{ll}
\mathbf{F}_{11}^{1, \varepsilon}(\omega, u)=\overline{\mathbf{F}_{22}^{1, \varepsilon}(\omega, u)}=\mathbf{H}^{a}\left(\omega, \frac{u}{\varepsilon^{s}}\right), & \mathbf{F}_{21}^{1, \varepsilon}(\omega, u)=\overline{\mathbf{F}_{12}^{1, \varepsilon}(\omega, u)}=\mathbf{H}^{b}\left(\omega, \frac{u}{\varepsilon^{s}}\right), \\
\mathbf{F}_{11}^{2, \varepsilon}(\omega, u)=\overline{\mathbf{F}_{22}^{2, \varepsilon}(\omega, u)}=\mathbf{G}^{a}\left(\omega, \frac{u}{\varepsilon^{s}}\right), & \mathbf{F}_{21}^{2, \varepsilon}(\omega, u)=\overline{\mathbf{F}_{12}^{2, \varepsilon}(\omega, u)}=\mathbf{G}^{b}\left(\omega, \frac{u}{\varepsilon^{s}}\right),
\end{array}
$$

to make the distinction in the forthcoming computations between the terms produce by the forward (resp. backward)-going propagating modes and forward (resp. backward)-going evanescent modes. As a result, we can write $\mathcal{P}^{\varepsilon, n}(\omega, z)$ as follows

$$
\mathcal{P}^{\varepsilon, n}(\omega, z)=\sum_{\left(i_{1}, \ldots, i_{n}\right) \in\{1,2\}^{n}} \int \cdots \int_{\Delta_{n}(z)} \prod_{m=1}^{n} d u_{m}\left[\prod_{m=1}^{n} \varepsilon^{i_{m} / 2-s} \mathbf{F}^{i_{m}, \varepsilon}\left(\omega, u_{m}\right)\right]\left[\begin{array}{c}
I d_{N} \\
\mathbf{0}
\end{array}\right]
$$

and

$$
\prod_{m=1}^{n} \varepsilon^{i_{m} / 2-s} \mathbf{F}^{i_{m}, \varepsilon}\left(\omega, u_{m}\right)\left[\begin{array}{c}
I d_{N} \\
\mathbf{0}
\end{array}\right]=\varepsilon^{\frac{1}{2} \sum_{m=1}^{n} i_{m}-n s}\left[\begin{array}{c}
\sum_{\left(p_{1}, \ldots, p_{n}\right) \in\{1,2\}^{n}} \prod_{m=1}^{n} \mathbf{F}_{p_{m}-\varepsilon, p_{m}}^{i_{m}, \varepsilon}\left(\omega, u_{m}\right) \\
\sum_{\left(q_{1}, \ldots, q_{n}\right) \in\{1,2\}^{n}} \prod_{m=1}^{n} \mathbf{F}_{q_{m-1}, q_{m}}^{i_{m}, \varepsilon}\left(\omega, u_{m}\right)
\end{array}\right],
$$

where $p_{0}=p_{n}=q_{n}=1$ and $q_{0}=2$. As we will see, only the component $i_{l}=1$ and $p_{l}=1(l=1, \ldots, n)$ in (45) has a nontrivial limit. All the other terms converge to 0 in probability.

Proposition 6.1 The series (43) is well defined and

$$
\forall \eta>0, \quad \lim _{\varepsilon \rightarrow 0} \mathbb{P}\left(\left\|\mathcal{P}^{\varepsilon}(\omega, z)-\left[\begin{array}{c}
X^{\varepsilon}(\omega, z) \\
0
\end{array}\right]\right\|_{\mathcal{M}_{N}(\mathbb{C}) \times \mathcal{M}_{N}(\mathbb{C})}>\eta\right)=0,
$$

where $\mathcal{P}^{\varepsilon}(\omega, z)$ is defined by (42), and $X^{\varepsilon}(\omega, z)$ is defined by

$$
X^{\varepsilon}(\omega, z)=\sum_{n=0}^{+\infty} X^{\varepsilon, n}(\omega, z),
$$

with

$$
X^{\varepsilon, n}(\omega, z)=\frac{1}{\varepsilon^{n(s-1 / 2)}} \int \cdots \int_{\Delta_{n}(z)} \prod_{m=1}^{n} \mathbf{H}^{a}\left(\omega, \frac{u_{m}}{\varepsilon^{s}}\right) d u_{m} .
$$

Let us remark that $X^{\varepsilon}(\omega, z)$ corresponds to the terms $i_{l}=1$ and $p_{l}=1(l=1, \ldots, n)$ in $(45)$. In view of $(25) X^{\varepsilon}(\omega, z)$ would correspond to the dynamic of a forward-going wave only, with no evanescent mode. Therefore, for this term we have

$$
\frac{d}{d z} X^{\varepsilon}(\omega, z)=\frac{1}{\varepsilon^{s-1 / 2}} \mathbf{H}^{a}\left(\omega, \frac{z}{\varepsilon^{s}}\right) X^{\varepsilon}(\omega, z) \quad \text { with } \quad X^{\varepsilon}(\omega, 0)=I d,
$$

so that for all $z \in[0, L]$

$$
\sum_{j, l=1}^{N(\omega)}\left|X_{j l}^{\varepsilon}(\omega, z)\right|^{2}=N(\omega),
$$

since $\mathbf{H}^{a}(\omega, z)$ is skew Hermitian, meaning that $X^{\varepsilon}(\omega, \cdot)$ is uniformly bounded. The following result deals with the asymptotic behavior of $X^{\varepsilon}(\omega, \cdot)$, and then concludes the proof of Theorem 2.1 according to [4, Theorem 3.1 pp. 27].

Proposition 6.2 For all $z \in[0, L]$, the family $\left(X^{\varepsilon}(\omega, z)\right)_{\varepsilon}$ converges in distribution on $\mathcal{M}_{N}(\mathbb{C})$ to

$$
D(\omega, z)=\operatorname{diag}\left(e^{i \sigma_{1, H}(\omega) B_{H}(z)}, \ldots, e^{i \sigma_{N, H}(\omega) B_{H}(z)}\right),
$$

with $\sigma_{j, H}(\omega)$ defined by (28), and where $B_{H}$ is a standard fractional Brownian motion with Hurst index $H=(2-\mathfrak{h}) / 2$, and defined on a probability space $(\tilde{\Omega}, \tilde{\mathcal{T}}, \tilde{\mathbb{P}})$.

The remaining of this section consists of proving Proposition 6.2 and Proposition 6.1. We start with the proof Proposition 6.2 because it allows us to illustrate all the important points arising in the proof of Proposition 6.1.

Let us note that we will prove in Proposition 6.1 (resp. Proposition 6.2) the convergence in distribution on $\mathcal{M}_{N}(\mathbb{C}) \times \mathcal{M}_{N}(\mathbb{C})\left(\right.$ resp. $\left.\mathcal{M}_{N}(\mathbb{C})\right)$ equipped with the weak topology. However, since the weak and the strong topology are the same on finite-dimensional vector spaces this strategy allows lighter notations without changing the result. 


\subsection{Proof of Proposition 6.2}

To prove the convergence of $\left(X^{\varepsilon}(\omega, z)\right)_{\varepsilon}$ we only have to focus on the convergence of its moments. In fact, $\left(X^{\varepsilon}(\omega, z)\right)_{\varepsilon}$ being a bounded family we directly have its tightness, that is

$$
\forall \eta>0, \quad \exists \mu>0 \quad \text { such that } \varlimsup_{\varepsilon \rightarrow 0} \mathbb{P}\left(\left|\left\langle X^{\varepsilon}(\omega, z), \lambda\right\rangle\right|^{2}>\mu\right) \leq \eta .
$$

Therefore, the computation of the moments allows us to characterize uniquely all the accumulation points. To compute the moments, we focus first on the first order moment as illustration (Proposition $6.3)$

$$
\mathbb{E}\left[\left\langle X^{\varepsilon}(\omega, z), \lambda\right\rangle\right]=\sum_{j, l=1}^{N} \mathbb{E}\left[X_{j l}^{\varepsilon}(\omega, z)\right] \overline{\lambda_{j l}}
$$

where $\lambda \in \mathcal{M}_{N}(\mathbb{C})$. Then, we investigate the arbitrary high order moments (Proposition 6.4)

$$
\begin{aligned}
\mathbb{E}\left[\left\langle X^{\varepsilon}(\omega, z), \lambda\right\rangle^{M_{1}} \overline{\left\langle X^{\varepsilon}(\omega, z), \lambda\right\rangle}{ }^{M_{2}}\right] & \\
=\sum_{p_{1}=1}^{M_{1}} \sum_{j_{1, p_{1}}, l_{1, p_{1}}=1}^{N} \sum_{p_{2}=1}^{M_{2}} \sum_{j_{2, p_{2}}, l_{2, p_{2}}=1}^{N} \mathbb{E}\left[\prod_{p_{1}=1}^{M_{1}} X_{j_{1, p_{1}} l_{1, p_{1}}}^{\varepsilon}(\omega, z) \prod_{p_{2}=1}^{M_{2}} \overline{X_{j_{2, p_{2}} l_{2, p_{2}}}^{\varepsilon}}(\omega, z)\right] & \times \prod_{p_{1}=1}^{M_{1}} \frac{\lambda_{j_{1, p_{1}} l_{1, p_{1}}}}{\prod_{p_{2}=1}^{M_{2}} \lambda_{j_{2, p_{2}} l_{2, p_{2}} .} .}
\end{aligned}
$$

\subsubsection{Proof of Proposition 6.2 : Moment of order one}

In this section we investigate the convergence of the expectation of $X^{\varepsilon}(\omega, z)$. This step is also useful to understand more easily the computations which are similar for the high order moments. Throughout this section let $(j, l) \in\{1, \ldots, N\}^{2}$ be two fixed indexes. According to (47), we have

$$
X_{j l}^{\varepsilon, n}(\omega, z)=\frac{i^{n} k^{2 n}(\omega)}{2^{n} \varepsilon^{n(s-1 / 2)}} \sum_{j_{1}, \ldots, j_{n-1}=1}^{N} \int \cdots \int_{\Delta_{n}(z)} \prod_{m=1}^{n} C_{j_{m-1} j_{m}}\left(\omega, u_{m} / \varepsilon^{s}\right) e^{i\left(\beta_{j_{m}}(\omega)-\beta_{j_{m-1}}(\omega)\right) u_{m} / \varepsilon^{s}} d u_{m}
$$

where $j_{0}=j$ and $j_{n}=l$.

Proposition 6.3 For all $(j, l) \in\{1, \ldots, N\}^{2}$, we have

$$
\lim _{\varepsilon \rightarrow 0} \mathbb{E}\left[X_{j l}^{\varepsilon}(\omega, z)\right]=\tilde{\mathbb{E}}\left[e^{i \sigma_{j, H}(\omega) B_{H}(z)} \delta_{j l}\right]
$$

where $\tilde{\mathbb{E}}$ is the expectation associated to the probability space on which the standard fractional Brownian motion $B_{H}$ is defined.

Proof (of Proposition 6.3) To compute the limit in $\varepsilon$ of $\mathbb{E}\left[X_{j l}^{\varepsilon}(\omega, z)\right]$, we have the two following lemmas.

Lemma 6.1 The series (46) is well defined, and we have for all $(j, l) \in\{1, \ldots, N(\omega)\}^{2}$

$$
\mathbb{E}\left[X_{j l}^{\varepsilon}(\omega, z)\right]=\mathbb{E}\left[\sum_{n=0}^{+\infty} X_{j l}^{\varepsilon, n}(\omega, z)\right]=\sum_{n=0}^{+\infty} \mathbb{E}\left[X_{j l}^{\varepsilon, n}(\omega, z)\right],
$$

and

$$
\lim _{\varepsilon \rightarrow 0} \mathbb{E}\left[X_{j l}^{\varepsilon}(\omega, z)\right]=\sum_{n=0}^{+\infty} \lim _{\varepsilon \rightarrow 0} \mathbb{E}\left[X_{j l}^{\varepsilon, n}(\omega, z)\right] .
$$

Proof (of Lemma 6.1) This lemma follows from a simple adaptation of the proof of the first point in Proposition 5.2. In fact, it suffices to show

$$
\sum_{n \geq 0} \sup _{\varepsilon \in(0,1)} \mathbb{E}\left[\left|X_{j l}^{\varepsilon, n}(\omega, z)\right|^{2}\right]^{1 / 2}<+\infty
$$


and we have

$$
\begin{aligned}
\mathbb{E}\left[\left|X_{j l}^{\varepsilon, n}(\omega, z)\right|^{2}\right] & \leq \frac{k^{4 n}(\omega)}{2^{2 n} \varepsilon^{2 n(s-1 / 2)}} \sum_{\substack{j_{1}^{1}, \ldots, j_{n-1}^{1}=1 \\
j_{1}^{2}, \ldots, j_{n-1}^{2}=1}}^{N} \int \cdots \int_{\Delta_{n}(z)} \int \cdots \int_{\Delta_{n}(z)} \prod_{m=1}^{n} d u_{m}^{1} d u_{m}^{2} \\
& \times\left|\mathbb{E}\left[\prod_{m=1}^{n} C_{j_{m-1}^{1} j_{m}^{1}}\left(\omega, u_{m}^{1} / \varepsilon^{s}\right) C_{j_{m-1}^{2} j_{m}^{2}}\left(\omega, u_{m}^{2} / \varepsilon^{s}\right)\right]\right| \\
& \leq \frac{C^{2 n}}{(n !)^{2}}
\end{aligned}
$$

where the $C_{j l}$ are defined by (17), which concludes the proof of the lemma.

Lemma 6.1 concerns the inversion between the expectation and the sum with respect to $n$, as well as the inversion between the limit in $\varepsilon$ and the sum. As a result, we have

$$
\lim _{\varepsilon \rightarrow 0} \mathbb{E}\left[X_{j l}^{\varepsilon}(\omega, z)\right]=\sum_{n=0}^{+\infty} \frac{i^{n} k^{2 n}(\omega)}{2^{n}} \sum_{j_{1}, \ldots, j_{n-1}=1}^{N} \lim _{\varepsilon \rightarrow 0} I_{j_{0}, \ldots, j_{n}}^{n, \varepsilon}(z),
$$

where

$$
I_{j_{0}, \ldots, j_{n}}^{n, \varepsilon}(z)=\frac{1}{\varepsilon^{n(s-1 / 2)}} \int \ldots \int_{\Delta_{n}(z)} \prod_{m=1}^{n} \frac{e^{i\left(\beta_{j_{m}}(\omega)-\beta_{j_{m-1}}(\omega)\right) u_{m} / \varepsilon^{s}}}{\sqrt{\beta_{j_{m-1}}(\omega) \beta_{j_{m}}(\omega)}} \mathbb{E}\left[\prod_{m=1}^{n} C_{j_{m-1} j_{m}}\left(\omega, u_{m} / \varepsilon^{s}\right)\right] d u_{1} \ldots d u_{n}
$$

with $j_{0}=j$ and $j_{n}=l$. However, form the Gaussian property of $\mathcal{B}_{\mathfrak{h}}$ and because $\Theta$ is an odd function the product in the expectation needs to contain an even number of terms, let say $n=2 n^{\prime}$, otherwise the expectation is 0 . Therefore, according to the second point of Proposition 5.2 we have

$$
\mathbb{E}\left[\prod_{m=1}^{2 n^{\prime}} C_{j_{m-1} j_{m}}\left(\omega, u_{m} / \varepsilon^{s}\right)\right] \underset{\varepsilon \rightarrow 0}{\sim} C_{\mathfrak{h}}^{n^{\prime}} \varepsilon^{n^{\prime} s \mathfrak{h}} \sum_{\mathcal{F}_{2 n^{\prime}}} \prod_{(\alpha, \gamma) \in \mathcal{F}_{2 n^{\prime}}} \frac{R_{j_{\alpha-1} j_{\alpha} j_{\gamma-1} j_{\gamma}}}{\left|u_{\alpha}-u_{\gamma}\right|^{\mathfrak{h}}}
$$

where the sum is over all the parings of $\left\{1, \ldots, 2 n^{\prime}\right\}$. As a result, for $s=1 /(2 H)$ with $H=(2-\mathfrak{h}) / 2$, the limit in $\varepsilon$ of the diagonal terms is

$$
\lim _{\varepsilon \rightarrow 0} I_{j, j, \ldots, j}^{2 n^{\prime}, \varepsilon}(z)=\left(\frac{C_{\mathfrak{h}} R_{j j j j}}{\beta_{j}^{2}(\omega)}\right)^{n^{\prime}} \int \ldots \int_{\Delta_{2 n^{\prime}}(z)} \sum_{\mathcal{F}_{2 n^{\prime}}} \prod_{(\alpha, \gamma) \in \mathcal{F}_{2 n^{\prime}}}\left|u_{\alpha}-u_{\gamma}\right|^{2 H-2} d u_{1} \ldots d u_{2 n^{\prime}} .
$$

However, we also have

$$
\sum_{\mathcal{F}_{2 n^{\prime}}} \prod_{(\alpha, \gamma) \in \mathcal{F}_{2 n^{\prime}}}\left|u_{\alpha}-u_{\gamma}\right|^{2 H-2}=c_{1, H}^{n^{\prime}} \tilde{\mathbb{E}}\left[\prod_{q=1}^{2 n^{\prime}} \int \frac{e^{i r_{q} u_{q}}}{\left|r_{q}\right|^{H-1 / 2}} w\left(d r_{q}\right)\right]
$$

where $w(d r)$ is a Gaussian white noise defined on a probability space $(\tilde{\Omega}, \tilde{\mathcal{T}}, \tilde{\mathbb{P}})$, and

$$
c_{1, H}=\Gamma(2 H-1) \sin (\pi H) / \pi \text {. }
$$

Therefore, we obtain

$$
\begin{aligned}
\lim _{\varepsilon \rightarrow 0} I_{j, j, \ldots, j}^{2 n^{\prime}, \varepsilon}(z) & =\left(C_{\mathfrak{h}} \frac{R_{j j j j}}{\beta_{j}^{2}(\omega)}\right)^{n^{\prime}} \frac{c_{1, H}^{n^{\prime}}}{\left(2 n^{\prime}\right) !} \tilde{\mathbb{E}}\left[\prod_{q=1}^{2 n^{\prime}} \int \frac{e^{i r_{q} z}-1}{i r_{q}\left|r_{q}\right|^{H-1 / 2}} w\left(d r_{q}\right)\right] \\
& =\left(C_{\mathfrak{h}} \frac{R_{j j j j}}{\beta_{j}^{2}(\omega)}\right)^{n^{\prime}} \frac{c_{1, H}^{n^{\prime}} c_{2, H}^{n^{\prime}}}{\left(2 n^{\prime}\right) !} \tilde{\mathbb{E}}\left[B_{H}^{2 n^{\prime}}(z)\right],
\end{aligned}
$$

with

$$
c_{2, H}=\pi /(H(2 H-1) \Gamma(2 H-1) \sin (H \pi))
$$


and

$$
B_{H}(z)=\frac{1}{c_{2, H}^{1 / 2}} \int \frac{e^{i r z}-1}{i r|r|^{H-1 / 2}} w(d r)
$$

is a standard fractional Brownian motion with Hurst index $H=(2-\mathfrak{h}) / 2$. As a result, we obtain

$$
\lim _{\varepsilon \rightarrow 0} I_{j, j, \ldots, j}^{2 n^{\prime}, \varepsilon}(z)=\tilde{\mathbb{E}}\left[\frac{\left(\tilde{\sigma}_{j, H}(\omega) B_{H}(z)\right)^{2 n^{\prime}}}{\left(2 n^{\prime}\right) !}\right] \quad \text { where } \quad \tilde{\sigma}_{j, H}^{2}(\omega)=\frac{C_{\mathfrak{h}} R_{j j j j}}{H(2 H-1) \beta_{j}^{2}(\omega)},
$$

and $R_{j l m n}$ is defined by (29). The following lemma deals with the offdiagonal terms, and shows that these terms converge to 0 as $\varepsilon \rightarrow 0$ because of the fast oscillating phase terms.

Lemma 6.2 If there exists $n_{0} \in\left\{1, \ldots, 2 n^{\prime}\right\}$ such that $j_{n_{0}-1} \neq j_{n_{0}}$, then

$$
\lim _{\varepsilon \rightarrow 0} I_{j_{0}, \ldots, j_{2 n^{\prime}}}^{n, \varepsilon}(z)=0
$$

Proof (of Lemma 6.2) According to the second point of Proposition 5.2 we have

$$
\begin{aligned}
I_{j_{0}, \ldots, j_{2 n^{\prime}}}^{n, \varepsilon}(z) & \underset{\varepsilon \rightarrow 0}{\sim} \prod_{m=1}^{2 n^{\prime}} \frac{C_{\mathfrak{h}}}{\sqrt{\beta_{j_{m-1}}(\omega) \beta_{j_{m}}(\omega)}} \sum_{\mathcal{F}_{2 n^{\prime}}} \int \cdots \int_{\Delta_{2 n^{\prime}}(z)} \prod_{m=1}^{2 n^{\prime}} d u_{m} \\
& \times \prod_{(\alpha, \gamma) \in \mathcal{F}_{2 n^{\prime}}} \frac{R_{j_{\alpha-1} j_{\alpha} j_{\gamma-1} j_{\gamma}}}{\left|u_{\alpha}-u_{\gamma}\right|^{\mathfrak{h}}} e^{i\left(\beta_{j_{\alpha}}(\omega)-\beta_{j_{\alpha-1}}(\omega)\right) u_{\alpha} / \varepsilon^{s}} e^{i\left(\beta_{j_{\gamma}}(\omega)-\beta_{j_{\gamma-1}}(\omega)\right) u_{\gamma} / \varepsilon^{s}} d u_{\alpha} d u_{\gamma} .
\end{aligned}
$$

For a fixed pairing $\mathcal{F}_{2 n^{\prime}}$ let us consider the couple $\left(\alpha_{0}, \gamma_{0}\right)$ involving $n_{0}$, let say $\alpha_{0}=n_{0}$. Using the fact that

$$
|u-v|^{-\mathfrak{h}}=c_{1, H} \int \frac{e^{i r(u-v)}}{|r|^{1-\mathfrak{h}}} d r
$$

we have

$$
\begin{aligned}
&\left|u_{\alpha_{0}}-u_{\gamma_{0}}\right|^{-\mathfrak{h}} e^{i\left(\beta_{j_{0}}(\omega)-\beta_{j_{\alpha_{0}-1}}(\omega)\right) u_{\alpha_{0}} / \varepsilon^{s}} e^{i\left(\beta_{j_{\gamma_{0}}}(\omega)-\beta_{j_{\gamma_{0}-1}}(\omega)\right) u_{\gamma_{0}} / \varepsilon^{s}} \\
&=c_{1, \mathfrak{h}} \int \frac{1}{|r|^{1-\mathfrak{h}}} e^{i u_{\alpha_{0}}\left(r+\left(\beta_{\alpha_{0}}(\omega)-\beta_{j_{\alpha_{0}-1}}(\omega)\right) / \varepsilon^{s}\right)} \\
& \times e^{-i u_{\gamma_{0}}\left(r-\left(\beta_{j_{0}}(\omega)-\beta_{j_{\gamma_{0}-1}}(\omega)\right) / \varepsilon^{s}\right)} d r .
\end{aligned}
$$

Using an integration by part in the variables $u_{\alpha_{0}}$ for

$$
e^{i u\left(r+\left(\beta_{\tilde{j}}(\omega)-\beta_{\tilde{l}}(\omega)\right) / \varepsilon^{s}\right)} \quad \text { with primitive } \quad \frac{e^{i u\left(r+\left(\beta_{\tilde{j}}(\omega)-\beta_{\tilde{l}}(\omega)\right) / \varepsilon^{s}\right)}-1}{i\left(r+\left(\beta_{\tilde{j}}(\omega)-\beta_{\tilde{l}}(\omega)\right) / \varepsilon^{s}\right)},
$$

we obtain

$$
\begin{aligned}
& \varlimsup_{\varepsilon \rightarrow 0}\left|I_{j_{0}, \ldots, j_{2 n^{\prime}}}^{n, \varepsilon}(z)\right| \leq \varlimsup_{\varepsilon \rightarrow 0} C_{1} \int_{\Delta_{2 n^{\prime}}^{(1)}(z)} \prod_{\substack{m=1 \\
m \neq \alpha_{0} \\
m \neq \gamma_{0}}}^{2 n^{\prime}} d u_{m} \prod_{\substack{(\alpha, \gamma) \in \mathcal{F}_{2 n^{\prime}} \\
(\alpha, \gamma) \neq\left(\alpha_{0}, \gamma_{0}\right)}}\left|u_{\alpha}-u_{\gamma}\right|^{-\mathfrak{h}} \\
& \times \int\left|\frac{e^{i u_{\alpha_{0}-1}\left(r+\left(\beta_{j_{\alpha_{0}}}(\omega)-\beta_{j_{\alpha_{0}-1}}(\omega)\right) / \varepsilon^{s}\right)}-1}{r+\left(\beta_{j_{\alpha_{0}}}(\omega)-\beta_{j_{\alpha_{0}-1}}(\omega)\right) / \varepsilon^{s}}\right| \frac{d r}{|r|^{1-\mathfrak{h}}} \\
& +\varlimsup_{\varepsilon \rightarrow 0} C_{2} \int_{\Delta_{2 n^{\prime}}^{(2)}(z)} \prod_{\substack{m=1 \\
m \neq \alpha_{0}+1 \\
m \neq \gamma_{0}}}^{2 n^{\prime}} d u_{m} \prod_{\substack{(\alpha, \gamma) \in \mathcal{F}_{2 n^{\prime}} \\
(\alpha, \gamma) \neq\left(\alpha_{0}+1, \gamma_{0}\right)}}\left|u_{\alpha}-u_{\gamma}\right|^{-\mathfrak{h}} \\
& \times \int\left|\frac{e^{i u_{\alpha_{0}}\left(r+\left(\beta_{j_{\alpha_{0}}}(\omega)-\beta_{j_{\alpha_{0}-1}}(\omega)\right) / \varepsilon^{s}\right)}-1}{r+\left(\beta_{j_{\alpha_{0}}}(\omega)-\beta_{j_{\alpha_{0}-1}}(\omega)\right) / \varepsilon^{s}}\right| \frac{d r}{|r|^{1-\mathfrak{h}}},
\end{aligned}
$$


where

$$
\begin{aligned}
& \Delta_{2 n^{\prime}}^{(1)}(z)=\left\{\left(u_{1}, \ldots, u_{\alpha_{0}-1}, u_{\alpha_{0}+1}, \ldots, u_{2 n^{\prime}}\right) \in[0, z]^{n-2},\right. \\
& \text { s.t } \left.u_{j} \leq u_{j-1} \quad \forall j \in\left\{2, \ldots, 2 n^{\prime}\right\} \backslash\left\{\alpha_{0}\right\}\right\} \\
& \Delta_{2 n^{\prime}}^{(2)}(z)=\left\{\left(u_{1}, \ldots, u_{\alpha_{0}}, u_{\alpha_{0}+2}, \ldots, u_{2 n^{\prime}}\right),\right. \\
& \text { s.t } \left.u_{j} \leq u_{j-1} \quad \forall j \in\left\{2, \ldots, 2 n^{\prime}\right\} \backslash\left\{\alpha_{0}+1\right\}\right\} .
\end{aligned}
$$

To conclude the proof Lemma 6.2, we have the following lemma.

Lemma 6.3 For all $a \neq 0$ and $u \neq 0$, we have

$$
\lim _{\varepsilon \rightarrow 0} \int \frac{\left|e^{i u\left(r-a / \varepsilon^{s}\right)}-1\right|}{\left|r-a / \varepsilon^{s}\right||r|^{1-\mathfrak{h}}} d r=0 .
$$

Proof (of Lemma 6.3) Let $\mu>0$ and $\eta>0$ be small parameters. We decompose the integral into three parts as follows

$$
\int \frac{\left|e^{i u\left(r-a / \varepsilon^{s}\right)}-1\right|}{\left|r-a / \varepsilon^{s}\right||r|^{1-\mathfrak{h}}} d r=\left(\int_{\left|r-a / \varepsilon^{s}\right|>\mu / \varepsilon^{s}}+\int_{\eta<\left|r-a / \varepsilon^{s}\right|<\mu / \varepsilon^{s}}+\int_{\left|r-a / \varepsilon^{s}\right|<\eta}\right) \frac{\left|e^{i u\left(r-a / \varepsilon^{s}\right)}-1\right|}{\left|r-a / \varepsilon^{s}\right||r|^{1-\mathfrak{h}}} d r .
$$

For the last integral, making the change of variable $r \rightarrow r+a / \varepsilon^{s} r$ we have

$$
\begin{aligned}
\int_{\left|r-a / \varepsilon^{s}\right|<\eta} \frac{\left|e^{i u\left(r-a / \varepsilon^{s}\right)}-1\right|}{\left|r-a / \varepsilon^{s}\right||r|^{2 H-1}} d r & =\int_{|r|<\eta} \frac{\left|e^{i u r}-1\right|^{2}}{|r|\left|r+a / \varepsilon^{s}\right|^{1-\mathfrak{h}}} d r \\
& \leq|u| \int_{|r|<\eta} \frac{d r}{\left|r+a / \varepsilon^{s}\right|^{1-\mathfrak{h}}} \\
& \leq|u| \varepsilon^{s(1-\mathfrak{h})} \int_{|r|<\eta} \frac{d r}{|| a\left|-\varepsilon^{s} \eta\right|^{1-\mathfrak{h}}} \\
& \leq C \varepsilon^{s(1-\mathfrak{h})} .
\end{aligned}
$$

For the second one, making the change of variable $r \rightarrow r / \varepsilon^{s}$ we have

$$
\begin{aligned}
\int_{\eta<\left|r-a / \varepsilon^{s}\right|<\mu / \varepsilon^{s}} \frac{\left|e^{i u\left(r-a / \varepsilon^{s}\right)}-1\right|^{2}}{\left|r-a / \varepsilon^{s}\right|^{2}|r|^{2 H-1}} d r & =\varepsilon^{s} \int_{\varepsilon^{s} \eta<\left|\varepsilon^{s} r-a\right|<\mu} \frac{\left|e^{i u\left(\varepsilon^{s} r-a\right) / \varepsilon^{s}}-1\right|}{\left|\varepsilon^{s} r-a\right||r|^{1-\mathfrak{h}}} d r \\
& =\varepsilon^{s(1-\mathfrak{h})} \int_{\varepsilon^{s} \eta<|r-a|<\mu} \frac{d r}{|r-a||r|^{1-\mathfrak{h}}} \\
& \leq \frac{e^{s(1-\mathfrak{h})}}{\left(|a|-\varepsilon^{s} \mu\right)^{1-\mathfrak{h}}} \int_{\varepsilon^{s} \eta<|u|<\mu} \frac{d r}{|u|} \\
& \leq C \varepsilon^{s(1-\mathfrak{h})} \log (1 / \varepsilon) .
\end{aligned}
$$

Finally, making the change of variable $r \rightarrow r / \varepsilon^{s}$, we have

$$
\begin{aligned}
\int_{\left|r-a / \varepsilon^{s}\right|>\mu / \varepsilon^{s}} \frac{\left|e^{i u\left(r-a / \varepsilon^{s}\right)}-1\right|}{\left|r-a / \varepsilon^{s}\right||r|^{1-\mathfrak{h}}} d r & =\varepsilon^{s(1-\mathfrak{h})} \int_{|r-a|>\mu} \frac{\left|e^{i u(r-a) \varepsilon^{s}}-1\right|}{|r-a||r|^{1-\mathfrak{h}}} d r \\
& \leq \varepsilon^{s(1-\mathfrak{h})} \int_{|r-a|>\mu} \frac{d r}{|r-a||r|^{1-\mathfrak{h}}}
\end{aligned}
$$

As a result, we obtain

$$
\begin{aligned}
\lim _{\varepsilon} \mathbb{E}\left[X_{j l}^{\varepsilon}(\omega, z)\right] & =\sum_{n^{\prime}=0}^{+\infty} \frac{\left(i k^{2}(\omega) \tilde{\sigma}_{j, H} / 2\right)^{2 n^{\prime}}}{\left(2 n^{\prime}\right) !} \tilde{\mathbb{E}}\left[B_{H}^{2 n^{\prime}}(z)\right] \delta_{j l} \\
& =\tilde{\mathbb{E}}\left[\sum_{n=0}^{+\infty} \frac{\left(i \sigma_{j, H} B_{H}(z)\right)^{n}}{n !}\right] \delta_{j l} \\
& =\tilde{\mathbb{E}}\left[e^{i \sigma_{j, H} B_{H}(z)} \delta_{j l}\right]
\end{aligned}
$$

which concludes the proof Proposition 6.3. 


\subsubsection{Proof of Proposition 6.2 : Arbitrary Order Moments}

To identify properly the limit in distribution of $X^{\varepsilon}(\omega, z)$ as $\varepsilon \rightarrow 0$ we need to identify all the moments

$$
\lim _{\varepsilon \rightarrow 0} \mathbb{E}\left[\prod_{p_{1}=1}^{M_{1}} X_{j_{1, p_{1}} l_{1, p_{1}}}^{\varepsilon}(\omega, z) \prod_{p_{2}=1}^{M_{2}} \overline{X_{j_{2, p_{2}} l_{2, p_{2}}}^{\varepsilon}(\omega, z)}\right] .
$$

However, as we will see the computations follow the ones of the first order moment. In the previous expression and in the forthcoming computations all the indexes with the subscript 2 correspond to the complex conjugate terms.

Proposition 6.4 For all $\left(j_{1,1}, \ldots, j_{1, M_{1}}, j_{2,1}, \ldots, j_{2, M_{2}}\right) \in\{1, \ldots, N\}^{M_{1}+M_{2}}$, we have

$$
\begin{aligned}
\lim _{\varepsilon \rightarrow 0} \mathbb{E}\left[\prod_{p_{1}=1}^{M_{1}} X_{j_{1, p_{1}} l_{1, p_{1}}}^{\varepsilon}(\omega, z)\right. & \left.\prod_{p_{2}=1}^{M_{2}} \frac{X_{j_{2, p_{2}} l_{2, p_{2}}}^{\varepsilon}(\omega, z)}{M_{1}}\right] \\
& =\tilde{\mathbb{E}}\left[\prod_{p_{1}=1}^{M_{1}} e^{i \sigma_{j_{1, p_{1}}, H}(\omega) B_{H}(z)} \delta_{j_{1, p_{1}} l_{1, p_{1}}} \prod_{p_{2}=1}^{M_{2}} e^{-i \sigma_{j_{2, p_{2}}, H}(\omega) B_{H}(z)} \delta_{j_{2, p_{2}} l_{2, p_{2}}}\right],
\end{aligned}
$$

where $B_{H}$ is defined by (54).

Proof (of Proposition 6.4) Using (51) we have

$$
\begin{aligned}
& \prod_{p_{1}=1}^{M_{1}} X_{j_{1, p_{1}} l_{1, p_{1}}}^{\varepsilon}(\omega, z) \prod_{p_{2}=1}^{M_{2}} \frac{X_{j_{2, p_{2}} l_{2, p_{2}}}^{\varepsilon}(\omega, z)}{M_{1}} \sum_{p_{1}=1}^{N} \sum_{p_{2}=1}^{M_{2}} \sum_{n_{1, p_{1}}=0}^{+\infty} \sum_{n_{2, p_{2}}=0}^{+\infty} \sum_{j_{1, p_{1}, 1}, \ldots, j_{1, p_{1}, n_{1, p_{1}-1}=1}}^{N} \mathbf{X}_{\mathbf{n}, \mathbf{j}}^{\varepsilon} \\
& \quad=\sum_{\mathbf{J}_{2, p_{2}, q_{2}, 1}, \ldots, j_{2, p_{2}, n_{2, p_{2}}-1}=1} \mathbf{X}_{\mathbf{n}, \mathbf{j}}^{\varepsilon}
\end{aligned}
$$

where

$$
\begin{aligned}
& \mathbf{X}_{\mathbf{n}, \mathbf{j}}^{\varepsilon}=\frac{i^{n_{1}-n_{2}} k^{2 n}(\omega)}{2^{n} \varepsilon^{n(s-1 / 2)}} \prod_{p_{1}=1}^{M_{1}} \int \cdots \int_{\Delta_{n_{1, p_{1}}}(z)} \prod_{p_{2}=1}^{M_{2}} \int \cdots \int_{\Delta_{n_{2, p_{2}}}(z)} \\
& \times \prod_{p_{1}=1}^{M_{1}} \prod_{m_{1, p_{1}}=1}^{n_{1, p_{1}}} C_{j_{1, p_{1}, m_{1, p_{1}}-1} j_{1, p_{1}, m_{1, p_{1}}}}\left(\omega, u_{1, p_{1}, m_{1, p_{1}}} / \varepsilon^{s}\right)
\end{aligned}
$$

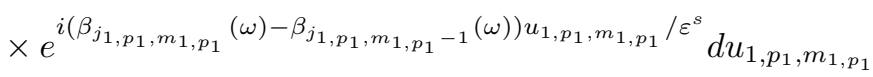

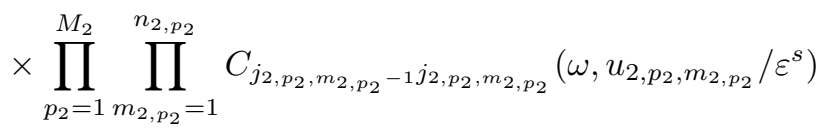

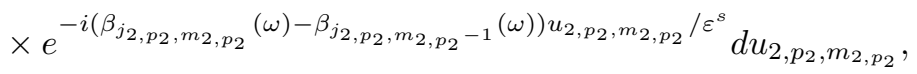

with

$$
n_{1}=\sum_{p_{1}=1}^{M_{1}} n_{1, p_{1}}, \quad n_{2}=\sum_{p_{2}=1}^{M_{2}} n_{2, p_{2}}, \quad \text { and } \quad n=n_{1}+n_{2} .
$$

Moreover, we have $j_{1, p_{1}, 0}=j_{1, p_{1}}, j_{1, p_{1}, n_{1, p_{1}}}=l_{1, p_{1}}, j_{2, p_{2}, 0}=j_{2, p_{2}}$, and $j_{2, p_{2}, n_{2, p_{2}}}=l_{2, p_{2}}$. To obtain the limit in $\varepsilon$ of the expectation of the previous expression, we need first to exchange the infinite sums with the expectation. To do so, we use an adapted version of Lemma 6.1.

Lemma 6.4 We have

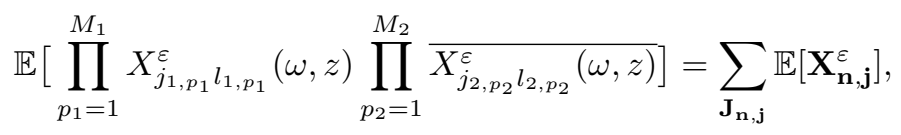


and

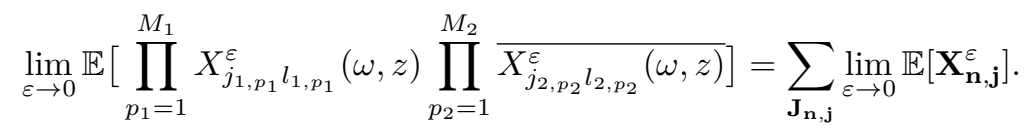

Proof (of Lemma 6.4) To prove this lemma, it suffices to show,

$$
\sum_{\mathbf{J}_{\mathbf{n}, \mathbf{j}}} \sup _{\varepsilon \in(0,1)} \mathbb{E}\left[\left|\mathbf{X}_{\mathbf{n}, \mathbf{j}}^{\varepsilon}\right|^{2}\right]^{1 / 2}<+\infty
$$

Following the proof of Lemma 6.1, we have

$$
\mathbb{E}\left[\left|\mathbf{X}_{\mathbf{n}, \mathbf{j}}^{\varepsilon}\right|^{2}\right] \leq \frac{k^{4 n}(\omega)}{2^{2 n}} \prod_{p_{1}=1}^{M_{1}} \frac{1}{\left(n_{1, p_{1}} !\right)^{2}} \prod_{p_{2}=1}^{M_{2}} \frac{1}{\left(n_{2, p_{2}} !\right)^{2}}\left|\mathbf{E}_{\mathbf{n}, \mathbf{j}}^{\varepsilon}\right|,
$$

where

$$
\begin{aligned}
& \mathbf{E}_{\mathbf{n}, \mathbf{j}}^{\varepsilon}=\frac{1}{\varepsilon^{2 n(s-1 / 2)}} \int_{[0, z]^{2 n}} \mathbb{E}\left[\prod_{p_{1}=1}^{M_{1}} \prod_{m_{1, p_{1}}=1}^{n_{1, p_{1}}} C_{j_{1, p_{1}, m_{1, p_{1}}-1} j_{1, p_{1}, m_{1, p_{1}}}}\left(\omega, u_{1, p_{1}, m_{1, p_{1}}}^{1} / \varepsilon^{s}\right)\right.
\end{aligned}
$$

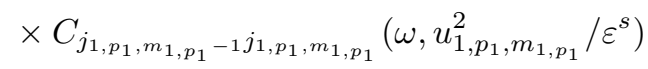

$$
\begin{aligned}
& \times \prod_{p_{2}=1}^{M_{2}} \prod_{m_{2, p_{2}}=1}^{n_{2, p_{2}}} C_{j_{2, p_{2}, m_{2, p_{2}}-1} j_{2, p_{2}, m_{2, p_{2}}}}\left(\omega, u_{2, p_{2}, m_{2, p_{2}}}^{1} / \varepsilon^{s}\right) \\
& \left.\times C_{j_{p_{2}, m_{2, p_{2}}-1} j_{p_{2}, m_{2, p_{2}}}}\left(\omega, u_{2, p_{2}, m_{2, p_{2}}}^{2} / \varepsilon^{s}\right)\right] \\
& \prod_{m_{1, p_{1}}=1}^{n_{1, p_{1}}} d u_{1, p_{1}, m_{1, p_{1}}}^{1} d u_{1, p_{1}, m_{1, p_{1}}}^{2} \prod_{m_{2, p_{2}}=1}^{n_{2, p_{2}}} d u_{2, p_{2}, m_{2, p_{2}}}^{1} d u_{2, p_{2}, m_{2, p_{2}}}^{2},
\end{aligned}
$$

so that for $s=1 /(2 H)$ we obtain by following the proof of the first point in Proposition 5.2

$$
\left|\mathbf{E}_{\mathbf{n}, \mathbf{j}}^{\varepsilon}\right| \leq C^{2 n} .
$$

Consequently, we have

$$
\sup _{\varepsilon \in(0,1)} \mathbb{E}\left[\left|\mathbf{X}_{\mathbf{n}, \mathbf{j}}^{\varepsilon}(\omega, z)\right|^{2}\right] \leq \prod_{p_{1}=1}^{M_{1}} \frac{C^{n_{1, p_{1}}}}{\left(n_{1, p_{1}} !\right)^{2}} \prod_{p_{2}=1}^{M_{2}} \frac{C^{n_{2, p_{2}}}}{\left(n_{2, p_{2}} !\right)^{2}}
$$

which concludes the proof of Lemma 6.4.

According to Lemma 6.4, to compute (55) it suffices to compute termwise $\lim _{\varepsilon \rightarrow 0} \mathbb{E}\left[\mathbf{X}_{\mathbf{n}, \mathbf{j}}^{\varepsilon}\right.$, which is done in the two following lemmas. The first one deals with the "diagonal" terms while the second one deals with the "offdiagonal" terms. In the first lemma, we need to have $n=2 n^{\prime}$ to obtain a nontrivial limit.

Lemma 6.5 If for all $\left(i, p_{i}, m_{i, p_{i}}\right) \in\{1,2\} \times\left\{1, \ldots, M_{i}\right\} \times\left\{1, \ldots, n_{i, p_{i}}\right\}$,

$$
j_{i, p_{i}, m_{p_{i}}}=j_{i, p_{i}}
$$

then we have

$$
\lim _{\varepsilon \rightarrow 0} \mathbb{E}\left[\mathbf{X}_{\mathbf{n}, \mathbf{j}}^{\varepsilon}\right]=\tilde{\mathbb{E}}\left[\prod_{p_{1}=1}^{M_{1}}\left[i \sigma_{j_{1, p_{1}}, H}(\omega) B_{H}(z)\right]^{n_{1, p_{1}}} \frac{1}{n_{1, p_{1}} !} \prod_{p_{2}=1}^{M_{2}}\left[-i \sigma_{j_{2, p_{2}}, H} B_{H}(z)\right]^{n_{2, p_{2}}} \frac{1}{n_{2, p_{2}} !}\right],
$$

with $\sigma_{j, H}(\omega)$ defined by $(28)$.

Lemma 6.6 If there exists $\left(i, p_{i}, m_{i, p_{i}}\right) \in\{1,2\} \times\left\{1, \ldots, M_{i}\right\} \times\left\{1, \ldots, n_{i, p_{i}}\right\}$ such that,

$$
j_{i, p_{i}, m_{i, p_{i}}} \neq j_{i, p_{i}, m_{i, p_{i}}-1}
$$

then we have

$$
\lim _{\varepsilon \rightarrow 0} \mathbb{E}\left[\mathbf{X}_{\mathbf{n}, \mathbf{j}}^{\varepsilon}\right]=0
$$


These two lemmas imply that

$$
\begin{aligned}
& \lim _{\varepsilon \rightarrow 0} \mathbb{E}\left[\prod_{p_{1}=1}^{M_{1}} X_{j_{1, p_{1}} l_{1, p_{1}}}^{\varepsilon}(\omega, z)\right. \prod_{p_{2}=1}^{M_{2}} \frac{X_{j_{2, p_{2}} l_{2, p_{2}}}^{\varepsilon}(\omega, z)}{X^{2}}=\sum_{\mathbf{J}_{\mathbf{n}, \mathbf{j}}} \lim _{\varepsilon \rightarrow 0} \mathbb{E}\left[\mathbf{X}_{\mathbf{n}, \mathbf{j}}^{\varepsilon}\right] \\
&=\tilde{\mathbb{E}}\left[\prod_{p_{1}=1}^{M_{1}} e^{i \sigma_{j_{1, p_{1}}, H}(\omega) B_{H}(z)} \delta_{j_{1, p_{1}} l_{1, p_{1}}} \prod_{p_{2}=1}^{M_{2}} e^{-i \sigma_{j_{2, p_{2}}, H}(\omega) B_{H}(z)} \delta_{j_{2, p_{2}} l_{2, p_{2}}}\right],
\end{aligned}
$$

which concludes the proof of Proposition 6.4.

Proof (of Lemma 6.5) Adapting the first point of Proposition 5.2, we have for $s=1 /(2 H)$ and $n=2 n^{\prime}$

$$
\begin{aligned}
& \lim _{\varepsilon \rightarrow 0} \mathbb{E}\left[\mathbf{X}_{\mathbf{n}, \mathbf{j}}^{\varepsilon}\right]=\frac{i^{n_{1}-n_{2}} k^{4 n^{\prime}}(\omega)}{2^{2 n^{\prime}}} \prod_{p_{1}=1}^{M_{1}}\left[\frac{R_{j_{1, p_{1}} j_{1, p_{1}} j_{1, p_{1}} j_{1, p_{1}}}^{1 / 2}}{\beta_{j_{1, p_{1}}}(\omega)}\right]^{n_{1, p_{1}}} \frac{1}{n_{1, p_{1}} !} \prod_{p_{2}=1}^{M_{2}}\left[\frac{R_{j_{2, p_{2}} j_{2, p_{2}} j_{2, p_{2}} j_{2, p_{2}}}^{1 / 2}}{\beta_{j_{2, p_{2}}}(\omega)}\right]^{n_{2, p_{2}}} \frac{1}{n_{2, p_{2}} !} \\
& \times \int_{[0, z]^{2 n^{\prime}}} \prod_{p_{1}=1}^{M_{1}} \prod_{m_{1, p_{1}}=1}^{n_{1, p_{1}}} d u_{1, p_{1}, q_{1}, m_{1, p_{1}}} \prod_{p_{2}=1}^{M_{2}} \prod_{m_{2, p_{2}}=1}^{n_{2, p_{2}}} d u_{2, p_{2}, m_{2, p_{2}}} \sum_{\mathcal{F}_{\mathbf{n}, \mathbf{j}}} \prod_{(\alpha, \gamma) \in \mathcal{F}_{\mathbf{n}, \mathbf{j}}} \frac{C_{\mathfrak{h}}}{\left|u_{\alpha}-u_{\gamma}\right|^{\mathfrak{h}}},
\end{aligned}
$$

where the sum is over all the pairings of $\mathbf{I}_{\mathbf{n}, \mathbf{j}}$ defined by

$$
\mathbf{I}_{\mathbf{n}, \mathbf{j}}=\left\{\left(i, p_{i}, m_{i, p_{i}}\right) \in\{1,2\} \times\left\{1, \ldots, M_{i}\right\} \times\left\{1, \ldots, n_{i, p_{i}}\right\}\right\} .
$$

Moreover, we have

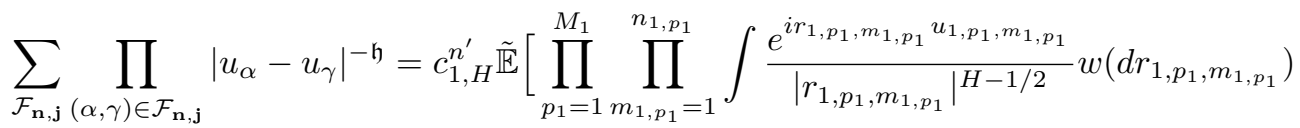

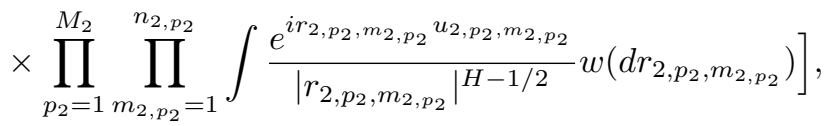

where $w(d r)$ is a Gaussian white noise on the probability space $(\tilde{\Omega}, \tilde{\mathcal{T}}, \tilde{\mathbb{P}}), c_{1, H}$ is given by $(52)$. Then, we finally have

$$
\begin{aligned}
\lim _{\varepsilon \rightarrow 0} \mathbb{E}\left[\mathbf{X}_{\mathbf{n}, \mathbf{j}}^{\varepsilon}\right] & =\tilde{\mathbb{E}}\left[\prod_{p_{1}=1}^{M_{1}}\left[\frac{i k^{2}(\omega)}{2 \beta_{j_{1, p_{1}}}(\omega)} \sqrt{c_{1, H} c_{2, H} C_{\mathfrak{h}} R_{j_{1, p_{1}} j_{1, p_{1}} j_{1, p_{1}} j_{1, p_{1}}}} B_{H}(z)\right]^{n_{1, p_{1}}} \frac{1}{n_{1, p_{1}} !}\right. \\
& \left.\times \prod_{p_{2}=1}^{M_{2}}\left[\frac{-i k^{2}(\omega)}{2 \beta_{j_{2, p_{2}}}(\omega)} \sqrt{c_{1, H} c_{2, H} C_{\mathfrak{h}} R_{j_{2, p_{2}} j_{2, p_{2}} j_{2, p_{2}} j_{2, p_{2}}}} B_{H}(z)\right]^{n_{2, p_{2}}} \frac{1}{n_{2, p_{2}} !}\right] .
\end{aligned}
$$

where $c_{2, H}$ is given by (53), and $B_{H}$ by (54).

Proof (of Lemma 6.6) First, we have

$$
\begin{aligned}
& \lim _{\varepsilon \rightarrow 0} \mathbb{E}\left[\mathbf{X}_{\mathbf{n}, \mathbf{j}}^{\varepsilon}\right]=\frac{k^{2 n}(\omega)}{2^{n}} \prod_{p_{1}=1}^{M_{1}} \int \cdots \int_{\Delta_{n_{1, p_{1}}}(z)} \prod_{m_{1, p_{1}}=1}^{n_{1, p_{1}}} d u_{1, p_{1}, m_{1, p_{1}}} \prod_{p_{2}=1}^{M_{2}} \int \cdots \int_{\Delta_{n_{1, p_{1}}}} \prod_{(z)} \prod_{m_{2, p_{2}}=1}^{n_{2, p_{2}}} d u_{2, p_{2}, m_{2, p_{2}}}
\end{aligned}
$$

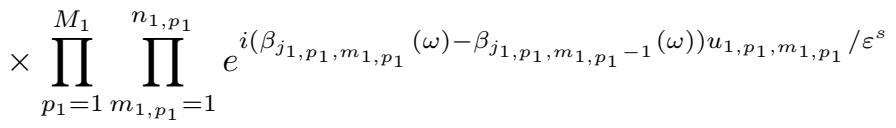

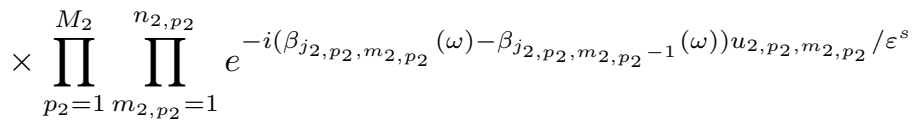

$$
\begin{aligned}
& \times \tilde{\mathbf{E}}_{\mathbf{n}, \mathbf{j}} \text {, }
\end{aligned}
$$


with

$$
\begin{aligned}
& \tilde{\mathbf{E}}_{\mathbf{n}, \mathbf{j}}=\lim _{\varepsilon \rightarrow 0} \frac{1}{\varepsilon^{n(s-1 / 2)}} \mathbb{E}\left[\prod_{p_{1}=1}^{M_{1}} \prod_{m_{1, p_{1}}=1}^{n_{1, p_{1}}} C_{j_{1, p_{1}, m_{1, p_{1}}-1} j_{1, p_{1}, m_{1, p_{1}}}}\left(\omega, u_{1, p_{1}, m_{1, p_{1}}} / \varepsilon^{s}\right)\right. \\
& \left.\times \prod_{p_{2}=1}^{M_{2}} \prod_{m_{2, p_{2}}=1}^{n_{2, p_{2}}} C_{j_{2, p_{2}, m_{2, p_{2}}-1} j_{2, p_{2}, m_{2, p_{2}}}}\left(\omega, u_{2, p_{2}, m_{2, p_{2}}} / \varepsilon^{s}\right)\right] \\
& =\sum_{\mathcal{F}_{\mathbf{n}, \mathbf{j}}} \prod_{(\alpha, \gamma) \in \mathcal{F}_{\mathbf{n}, \mathbf{j}}} \frac{C_{\mathfrak{h}}}{\left|u_{\alpha}-u_{\gamma}\right|^{\mathfrak{h}}} \frac{R_{j_{\alpha-(0,0,1)} j_{\alpha} j_{\gamma-(0,0,1)} j_{\gamma}}}{\sqrt{\beta_{j_{\alpha-(0,0,1)}}(\omega) \beta_{j_{\alpha}}(\omega) \beta_{j_{\gamma-(0,0,1)}}(\omega) \beta_{j_{\gamma}}(\omega)}},
\end{aligned}
$$

by adapting the proof of the second point in Proposition 5.2, and where the sum is over all the pairings of $\mathbf{I}_{\mathbf{n}, \mathbf{j}}$ defined by (56). Let us fixe a pairing $\mathcal{F}_{\mathbf{n}, \mathbf{j}}$ and denote $\mathbf{i}_{0}=\left(i, p_{i}, m_{i, p_{i}}\right)$ given in the statement of the lemma such that

$$
j_{i, p_{i}, m_{i, p_{i}}} \neq j_{i, p_{i}, m_{p_{i}}-1} .
$$

Following the proof of Lemma 6.2 and using Lemma 6.3, we obtain

$$
\begin{aligned}
\lim _{\varepsilon \rightarrow 0} \frac{1}{\varepsilon^{n(s-1 / 2)}} & \int \cdots \int_{\Delta_{i, p_{i}}(z)} \prod_{m_{i, p_{i}}=1}^{n_{i, p_{i}}} d u_{\alpha_{0}} \\
& \times \prod_{(\alpha, \gamma) \in \mathcal{F}_{\mathbf{n}, \mathbf{j}}}\left|u_{\alpha}-u_{\gamma}\right|^{-\mathfrak{h}} e^{i\left(\beta_{j_{\alpha}}(\omega)-\beta_{j_{\alpha-1}}(\omega)\right) u_{\alpha} / \varepsilon^{s}} e^{i\left(\beta_{j_{\gamma}}(\omega)-\beta_{j_{\gamma-1}}(\omega)\right) u_{\gamma} / \varepsilon^{s}}=0
\end{aligned}
$$

and then

$$
\lim _{\varepsilon \rightarrow 0} \mathbb{E}\left[\mathbf{X}_{\mathbf{n}, \mathbf{j}}^{\varepsilon}\right]=0
$$

since all the pairings $\mathcal{F}_{\mathbf{n}, \mathbf{j}}$ contain a term $\left(\alpha_{0}, \gamma_{0}\right)$ involving $\mathbf{i}_{0}$. That concludes the proof of Lemma 6.6 and at the same time the one of Proposition 6.4.

\subsection{Proof of Proposition 6.1}

The first point of the proposition follows the idea of Lemma 6.1, and for the second point (the convergence in probability) we only need to prove that

$$
\lim _{\varepsilon \rightarrow 0} \mathbb{E}\left[\left|\left\langle\mathcal{P}^{\varepsilon}(\omega, z)-\left[\begin{array}{c}
X^{\varepsilon}(\omega, z) \\
0
\end{array}\right],\left[\begin{array}{c}
\lambda_{1} \\
\lambda_{2}
\end{array}\right]\right\rangle\right|\right]=0
$$

thanks to the Markov's inequality, where

$$
\left\langle\mathcal{P}^{\varepsilon}(\omega, z)-\left[\begin{array}{c}
X^{\varepsilon}(\omega, z) \\
0
\end{array}\right],\left[\begin{array}{c}
\lambda_{1} \\
\lambda_{2}
\end{array}\right]\right\rangle=\sum_{j, l=1}^{N}\left(\mathbf{P}_{j l}^{a, \varepsilon}(\omega, z)-X_{j l}^{\varepsilon}(\omega, z)\right) \overline{\lambda_{1, j l}}+\mathbf{P}_{j l}^{b, \varepsilon}(\omega, z) \overline{\lambda_{2, j l}} .
$$

However, both points need the following lemma.

Lemma 6.7 We have

$$
\sum_{n \geq 0} \sup _{\varepsilon \in(0,1)} \mathbb{E}\left[\left|\mathcal{P}_{j l}^{1, \varepsilon, n}(\omega, z)\right|^{2}\right]^{1 / 2}+\mathbb{E}\left[\left|\mathcal{P}_{j l}^{2, \varepsilon, n}(\omega, z)\right|^{2}\right]^{1 / 2}<+\infty .
$$

According to this result and the proof of Lemma 6.1, we have

$$
\left.\lim _{\varepsilon \rightarrow 0} \mathbb{E}\left[\mid \mathbf{P}_{j l}^{a, \varepsilon}(\omega, z)-X_{j l}^{\varepsilon}(\omega, z)\right) \mid\right] \leq \sum_{n=0}^{+\infty} \lim _{\varepsilon \rightarrow 0} \mathbb{E}\left[\left|\mathcal{P}_{j l}^{1, \varepsilon, n}(\omega, z)-X_{j l}^{\varepsilon, n}(\omega, z)\right|^{2}\right]^{1 / 2}
$$

and

$$
\lim _{\varepsilon \rightarrow 0} \mathbb{E}\left[\left|\mathbf{P}_{j l}^{b, \varepsilon}(\omega, z)\right|\right] \leq \sum_{n=0}^{+\infty} \lim _{\varepsilon \rightarrow 0} \mathbb{E}\left[\left|\mathcal{P}_{j l}^{2, \varepsilon, n}(\omega, z)\right|^{2}\right]^{1 / 2}
$$


Proof (of Lemma 6.7) The proof of this result consists of adapting the one of Lemma 6.1. In fact, according to (44) and following the proof of the first point in Proposition 5.2 the terms

$$
\mathbb{E}\left[\left|\mathcal{P}_{j l}^{1, \varepsilon, n}(\omega, z)\right|^{2}\right] \quad \text { and } \quad \mathbb{E}\left[\left|\mathcal{P}_{j l}^{2, \varepsilon, n}(\omega, z)\right|^{2}\right]
$$

can be bounded by sums and products of terms of the form

$$
\int_{0}^{z} \int_{0}^{z}|u-v|^{-\mathfrak{h}} d u d v<+\infty
$$

and

$$
\frac{2}{\varepsilon^{2 s-3 / 2}} \int_{0}^{+\infty} d w e^{-\beta_{l}(\omega)|w|} \int_{0}^{z} \int_{0}^{u}\left|\frac{u}{\varepsilon^{s}}+w-\frac{v}{\varepsilon^{s}}\right|^{-\mathfrak{h}} d u d v \leq \frac{\sqrt{\varepsilon} C}{\beta_{l}(\omega)} \int_{0}^{z} \int_{0}^{z}|u-v|^{-\mathfrak{h}} d u d v<+\infty
$$

coming from $\mathbf{H}^{a}, \mathbf{H}^{b}, \mathbf{G}^{a}$, and $\mathbf{G}^{b}$ defined by (22). Let us remark that we get ride of the $w$ in the right hand side of (59) using the fact $w \rightarrow\left|u-v+\varepsilon^{s} w\right|^{-\mathfrak{h}}$ is a decreasing function with respect to $|w|$. Moreover, we have to remark that the infinite sums in the definition of $\mathbf{G}^{a}$, and $\mathbf{G}^{b}$ give rise to finite terms in all these estimates. In fact, thanks to (59), these infinite sums involve

$$
\sum_{l \geq N(\omega)+1} \frac{1}{\beta_{l}^{2}(\omega)}<+\infty
$$

since we consider a planar waveguide. Finally, with all these estimates we obtain

$$
\sum_{n \geq 0} \sup _{\varepsilon \in\left(0, \varepsilon_{0}\right)} \mathbb{E}\left[\left|\mathcal{P}_{j l}^{1, \varepsilon, n}(\omega, z)\right|^{2}\right]^{1 / 2}+\mathbb{E}\left[\left|\mathcal{P}_{j l}^{2, \varepsilon, n}(\omega, z)\right|^{2}\right]^{1 / 2} \leq \sum_{n \geq 0} \frac{C_{1}^{n} \sqrt{(2 n-1) ! !}}{n !} \leq \sum_{n \geq 0} \frac{C_{2}^{n}}{\sqrt{n !}}<+\infty
$$

which concludes the proof of Lemma 6.7 .

Now, to compute (57) and (58), we remark that according to (44) and Proposition 5.2, the terms

$$
\mathbb{E}\left[\left|\mathcal{P}_{j l}^{1, \varepsilon, n}(\omega, z)-X_{j l}^{\varepsilon, n}(\omega, z)\right|^{2}\right] \quad \text { and } \quad \mathbb{E}\left[\left|\mathcal{P}_{j l}^{2, \varepsilon, n}(\omega, z)\right|^{2}\right]
$$

can be expressed by sums and products of terms of the form

$$
|u-v|^{-\mathfrak{h}} e^{i\left(\beta_{l_{1}}(\omega)-\nu \beta_{j_{1}}(\omega)\right) u / e^{s}} e^{i\left(\beta_{l_{2}}(\omega)+\beta_{j_{2}}(\omega)\right) v / e^{s}},
$$

where $\nu \in\{-1,1\}$ and

$$
\begin{aligned}
\frac{1}{\varepsilon^{2 s-3 / 2}} & \int d w e^{i \beta_{l_{1}}(\omega) w} e^{-\beta_{l^{\prime}}(\omega)|w|} \\
& \times\left|u-v+\varepsilon^{s} w\right|^{-\mathfrak{h}} e^{i\left(\beta_{l_{1}}(\omega)-\nu_{1} \beta_{j_{1}}(\omega)\right) u / e^{s}} e^{i\left(\beta_{l_{2}}(\omega)-\nu_{2} \beta_{j_{2}}(\omega)\right) v / e^{s}},
\end{aligned}
$$

where $\left(\nu_{1}, \nu_{2}\right) \in\{-1,1\}^{2}$. The contribution of these two terms is 0 in the limit $\varepsilon \rightarrow 0$. It is easy to see according to (59) that the contribution of the second term is 0 in the limit $\varepsilon \rightarrow 0$. For the first one, it suffices to follows exactly the proof of Lemma 6.2. Consequently, we have

$$
\lim _{\varepsilon \rightarrow 0} \mathbb{E}\left[\left|\mathcal{P}_{j l}^{1, \varepsilon, n}(\omega, z)-X_{j l}^{\varepsilon, n}(\omega, z)\right|^{2}\right]=0 \quad \text { and } \quad \lim _{\varepsilon \rightarrow 0} \mathbb{E}\left[\left|\mathcal{P}_{j l}^{2, \varepsilon, n}(\omega, z)\right|^{2}\right]=0,
$$

which concludes the proof of Proposition 6.1.

\section{Proof of Theorem 3.1}

First of all, let us remark that according to Proposition 6.1, we have

$$
\lim _{\varepsilon \rightarrow 0} \mathbb{P}\left(\sup _{t, x}\left|p_{p r}\left(t, x, L / \varepsilon^{s}\right)-\tilde{p}_{p r}\left(t, x, L / \varepsilon^{s}\right)\right|>\eta\right)=0,
$$


where $p_{p r}$ is defined by (30), so that according to [4, Theorem 3.1 pp. 27] we just have to prove Theorem 3.1 by replacing $p_{p r}$ with

$$
\begin{aligned}
\tilde{p}_{p r}\left(t, x, \frac{L}{\varepsilon^{s}}\right)=\frac{1}{4 \pi \varepsilon^{q}} \int d \omega e^{-i \omega t} \widehat{f}\left(\frac{\omega-\omega_{0}}{\varepsilon^{q}}\right) & \sum_{j, l=1}^{N(\omega)} \sqrt{\frac{\beta_{l}(\omega)}{\beta_{j}(\omega)}} X_{j l}^{\varepsilon}(\omega, L) \\
& \times e^{i \beta_{j}(\omega) L / \varepsilon^{s}} e^{-i \beta_{l}(\omega) L_{S}} \phi_{j}(x)\left\langle\phi_{l}, \Psi\right\rangle_{L^{2}(0, d)} .
\end{aligned}
$$

Second, let us remark that $\mathcal{C}\left([-T, T] \times(0, d), \mathbb{C}^{N\left(\omega_{0}\right)}\right)$ equipped with the supremum norm on $[-T, T] \times$ $(0, d)$ is a separable Banach space, so that the tightness and the relative compactness are the same (see [4, Theorem 5.2 pp. 60]). Consequently, according to the Arzelà-Ascoli theorem, we only need to prove the following result.

Lemma 7.1 We have

$$
\lim _{M \rightarrow+\infty} \varlimsup_{\varepsilon \rightarrow 0} \mathbb{P}\left(\sup _{u, x} \sum_{j=1}^{N\left(\omega_{0}\right)}\left|\tilde{p}_{j, p r}^{\varepsilon}(u, x, L)\right|>M\right)=0,
$$

and for all $\eta>0$

$$
\lim _{\tau \rightarrow 0} \varlimsup_{\varepsilon \rightarrow 0} \mathbb{P}\left(\sup _{\left|x_{1}-x_{2}\right|+\left|u_{1}-u_{2}\right| \leq \tau} \sum_{j=1}^{N\left(\omega_{0}\right)}\left|\tilde{p}_{j, p r}^{\varepsilon}\left(u_{1}, x_{1}, L\right)-\tilde{p}_{j, p r}^{\varepsilon}\left(u_{2}, x_{2}, L\right)\right|>\eta\right)=0 .
$$

Consequently, using that the family $\left(\tilde{p}_{p r}\left(\cdot, \cdot, L / \varepsilon^{s}\right)\right)_{\varepsilon}$ is uniformly bounded, we just have to characterize all the possible limits through their moments as is done in the two following lemmas.

Lemma 7.2 We have

$$
\begin{aligned}
& \lim _{\varepsilon \rightarrow 0} \mathbb{E}\left[\prod_{m_{1}=1}^{\gamma_{1}} \tilde{p}_{\tilde{j}_{1, m_{1}}, p r}^{\varepsilon}\left(u_{1, m_{1}}, x_{1, m_{1}}, L\right) \prod_{m_{2}=1}^{\gamma_{2}} \frac{\overline{\tilde{p}_{j_{2, m_{2}}, p r}^{\varepsilon}}\left(u_{2, m_{2}}, x_{2, m_{2}}, L\right)}{\boldsymbol{\gamma}_{1}}\right] \\
& =\tilde{\mathbb{E}}\left[\prod_{m_{1}=1}^{\gamma_{1}} p_{\tilde{j}_{2, m_{2}}, p r}^{0}\left(u_{1, m_{1}}, x_{1, m_{1}}, L\right) \prod_{m_{2}=1}^{\gamma_{2}} \overline{p_{\tilde{j}_{2, m_{2}}, p r}^{0}\left(u_{2, m_{2}}, x_{2, m_{2}}, L\right)}\right],
\end{aligned}
$$

for all $\left(\gamma_{1}, \gamma_{2}\right) \in\left(\mathbb{N}^{*}\right)^{2},\left(u_{n, m}\right) \in[-T, T]^{\gamma_{1}+\gamma_{2}},\left(x_{n, m}\right) \in(0, d)^{\gamma_{1}+\gamma_{2}}$, and $\left(\tilde{j}_{n, m}\right) \in\left\{1, \ldots, N\left(\omega_{0}\right)\right\}^{\gamma_{1}+\gamma_{2}}$. Here, $p_{j, p r}^{0}$ is defined by (39) and $\tilde{\mathbb{E}}$ is the expectation associated to the probability space on which the standard fractional Brownian motion is defined.

Proof (of Lemma 7.1) This lemma is a direct consequence of (48). For the first point we have

$$
\varlimsup_{\varepsilon \rightarrow 0} \mathbb{E}\left[\sup _{j, u, x}\left|p_{j, p r}^{\varepsilon}(u, x, L)\right|\right] \leq C_{N\left(\omega_{0}\right)} \int|\widehat{f}(h)| d h<+\infty,
$$

in addition to the Markov's inequality. For the second point, in the same way and using the regularity of the eigenvectors $\phi_{j}$ we have for all $\tau>0$

$$
\varlimsup_{\varepsilon \rightarrow 0} \mathbb{E}\left[\sup _{j} \sup _{\left|x_{1}-x_{2}\right|+\left|u_{1}-u_{2}\right| \leq \tau}\left|p_{j, p r}^{\varepsilon}\left(u_{1}, x_{1}, L\right)-p_{j, p r}^{\varepsilon}\left(u_{2}, x_{2}, L\right)\right| \leq C_{N\left(\omega_{0}\right)} \tau \int|h \widehat{f}(h)| d h,\right.
$$

which concludes the proof of Lemma 7.1.

Proof (of Lemma 7.2) Expending the product

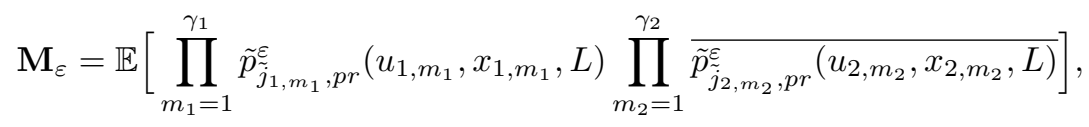


according to the definition (38) (with $X^{\varepsilon}(\omega, L)$ instead of $\mathbf{P}^{a, \varepsilon}(\omega, L)$ ), gives

$$
\begin{aligned}
& \mathbf{M}_{\varepsilon}=\frac{1}{(4 \pi)^{\gamma_{1}+\gamma_{2}}} \sum_{\substack{1 \leq m_{1} \leq \gamma_{1} \\
1 \leq m_{2} \leq \gamma_{2}}} \sum_{\substack{1 \leq j_{1, m_{1}}, l_{1, m_{1}} \leq N\left(\omega_{0}\right) \\
1 \leq j_{2, m_{2},}, l_{2, m_{2}} \leq N\left(\omega_{0}\right)}} \prod_{m_{1}, m_{2}} \sqrt{\frac{\beta_{l_{1, m_{1}}}\left(\omega_{0}\right) \beta_{l_{2, m_{2}}}\left(\omega_{0}\right)}{\beta_{j_{1, m_{1}}}\left(\omega_{0}\right) \beta_{j_{2, m_{2}}}\left(\omega_{0}\right)}} \\
& \times e^{i\left(\beta_{j_{1, m_{1}}}\left(\omega_{0}\right)-\beta_{\tilde{j}_{1, m_{1}}}\left(\omega_{0}\right)\right) L / \varepsilon^{s}} e^{-i\left(\beta_{j_{2, m_{2}}}\left(\omega_{0}\right)-\beta_{\tilde{j}_{2, m_{2}}}\left(\omega_{0}\right)\right) L / \varepsilon^{s}} \\
& \times e^{i\left(\beta_{\tilde{j}_{1, m_{1}}}\left(\omega_{0}\right)-\beta_{l_{1, m_{1}}}\left(\omega_{0}\right)\right) L_{S}} e^{-i\left(\beta_{\tilde{j}_{2, m_{2}}}\left(\omega_{0}\right)-\beta_{l_{2, m_{2}}}\left(\omega_{0}\right)\right) L_{S}} \\
& \times \phi_{j_{1, m_{1}}}\left(x_{1, m_{1}}\right) \phi_{l_{1, m_{1}}}\left(x_{0}\right) \phi_{j_{2, m_{2}}}\left(x_{2, m_{2}}\right) \phi_{l_{2, m_{2}}}\left(x_{0}\right) \\
& \times \int \cdots \int \prod_{m_{1}, m_{2}} d h_{1, m_{1}} d h_{2, m_{2}} \widehat{f}\left(h_{1, m_{1}}\right) \overline{\widehat{f}\left(h_{2, m_{2}}\right)} e^{-i\left(h_{1, m_{1}} u_{1, m_{1}}-h_{2, m_{2}} u_{2, m_{2}}\right)} \\
& \times e^{i h_{1, m_{1}}\left(\beta_{j_{1, m_{1}}}^{\prime}\left(\omega_{0}\right)-\beta_{\tilde{j}_{1, m_{1}}}^{\prime}\left(\omega_{0}\right)\right) L / \varepsilon^{s-q}} e^{-i h_{2, m_{2}}\left(\beta_{j_{2, m_{2}}}^{\prime}\left(\omega_{0}\right)-\beta_{\dot{j}_{2, m_{2}}}^{\prime}\left(\omega_{0}\right)\right) L / \varepsilon^{s-q}} \\
& \times e^{i\left(\Phi_{j_{1, m_{1}}}^{\varepsilon}\left(h_{1, m_{1}}\right)-\Phi_{j_{2, m_{2}}}^{\varepsilon}\left(h_{2, m_{2}}\right)\right) L / \varepsilon^{s-2 q}} \\
& \times \mathbb{E}\left[\prod_{m_{1}, m_{2}} X_{j_{1, m_{1}} l_{1, m_{1}}}^{\varepsilon}\left(\omega_{0}+\varepsilon^{q} h_{1, m_{1}}, L\right) \overline{X_{j_{2, m_{2}} l_{2, m_{2}}}^{\varepsilon}\left(\omega_{0}+\varepsilon^{q} h_{2, m_{2}}, L\right)}\right] .
\end{aligned}
$$

According to Proposition 6.4 (with slight adaptations regarding the frequency) we have

$$
\begin{aligned}
\lim _{\varepsilon \rightarrow 0} \mathbb{E} & {\left[\prod_{m_{1}, m_{2}} X_{j_{1, m_{1}} l_{1, m_{1}}}^{\varepsilon}\left(\omega_{0}+\varepsilon^{q} h_{1, m_{1}}, L\right) \overline{X_{j_{2, m_{2}} l_{2, m_{2}}}^{\varepsilon}\left(\omega_{0}+\varepsilon^{q} h_{2, m_{2}}, L\right)}\right] } \\
& =\tilde{\mathbb{E}}\left[\prod_{m_{1}, m_{2}} D_{j_{1, m_{1}} j_{1, m_{1}}}\left(\omega_{0}, L\right) \overline{D_{j_{2, m_{2}} j_{2, m_{2}}}\left(\omega_{0}, L\right)}\right] \prod_{m_{1}, m_{2}} \delta_{j_{1, m_{1}} l_{1, m_{1}}} \delta_{j_{2, m_{2}} l_{2, m_{2}}} .
\end{aligned}
$$

Moreover, using the Riemann-Lebesgue lemma and the terms of the form $e^{i h\left(\beta_{j}^{\prime}\left(\omega_{0}\right)-\beta_{\tilde{j}}^{\prime}\left(\omega_{0}\right)\right) L / \varepsilon^{s-q}}$ in $\mathbf{M}_{\varepsilon}$, the only nontrivial term in the limit $\varepsilon \rightarrow 0$ is obtained for $j_{1, m_{1}}=\tilde{j}_{1, m_{1}}$ and $j_{2, m_{2}}=\tilde{j}_{2, m_{2}}$. Therefore, in the case $q>s / 2$, we have

$$
\begin{aligned}
\lim _{\varepsilon \rightarrow 0} \mathbf{M}_{\varepsilon}=\tilde{\mathbb{E}}\left[\prod_{m_{1}=1}^{\gamma_{1}} \frac{D_{\tilde{j}_{1, m_{1}} \tilde{j}_{1, m_{1}}}\left(\omega_{0}, L\right)}{2}\right. & f\left(u_{1, m_{1}}\right) \phi_{\tilde{j}_{1, m_{1}}}\left(x_{1, m_{1}}\right) \phi_{\tilde{j}_{1, m_{1}}}\left(x_{0}\right) \\
& \left.\times \prod_{m_{2}=1}^{\gamma_{2}} \frac{\overline{D_{\tilde{j}_{2, m_{2}} \tilde{j}_{2, m_{2}}}\left(\omega_{0}, L\right)}}{2} f\left(u_{2, m_{2}}\right) \phi_{\tilde{j}_{2, m_{2}}}\left(x_{2, m_{2}}\right) \phi_{\tilde{j}_{2, m_{2}}}\left(x_{0}\right)\right],
\end{aligned}
$$

and in the case $q=s / 2$

$$
\begin{aligned}
\lim _{\varepsilon \rightarrow 0} \mathbf{M}_{\varepsilon}=\tilde{\mathbb{E}}\left[\prod_{m_{1}=1}^{\gamma_{1}}\right. & \frac{D_{\tilde{j}_{1, m_{1}} \tilde{j}_{1, m_{1}}}\left(\omega_{0}, L\right)}{2} K_{\tilde{j}_{1, m_{1}}, L} * f\left(u_{1, m_{1}}\right) \phi_{\tilde{j}_{1, m_{1}}}\left(x_{1, m_{1}}\right) \phi_{\tilde{j}_{1, m_{1}}}\left(x_{0}\right) \\
& \left.\times \prod_{m_{2}=1}^{\gamma_{2}} \frac{\overline{D_{\tilde{j}_{2, m_{2}} \tilde{j}_{2, m_{2}}}\left(\omega_{0}, L\right)}}{2} \overline{K_{\tilde{j}_{2, m_{2}}, L} * f\left(u_{2, m_{2}}\right)} \phi_{\tilde{j}_{2, m_{2}}}\left(x_{2, m_{2}}\right) \phi_{\tilde{j}_{2, m_{2}}}\left(x_{0}\right)\right] .
\end{aligned}
$$

Finally, the case $q \in(0, s / 2)$ is a consequence of the stationary phase method, which therefore concludes the proof of Lemma 7.2 .

\section{Proof of Theorem 3.2}

The proof of this theorem follows closely the one of Theorem 3.1. Using (61) we just need to prove Theorem 3.2 for $\tilde{p}_{p r}$ instead of $p_{p r}$. The tightness of the family $\left(\tilde{p}_{j, p r}^{\varepsilon}(\cdot, L)\right)_{j, \varepsilon}$ follows the same lines, and for the identification of the moments we also have using Proposition 6.4 (with slight adaptations regarding the frequency) for all $\left(\gamma_{1}, \gamma_{2}\right) \in\left(\mathbb{N}^{*}\right)^{2},\left(t_{n, m}\right) \in[-T, T]^{\gamma_{1}+\gamma_{2}}$, and $\left(\tilde{j}_{n, m}\right) \in(0, d)^{\gamma_{1}+\gamma_{2}}$

$$
\begin{aligned}
\lim _{\varepsilon \rightarrow 0} \mathbb{E}\left[\prod_{m_{1}=1}^{\gamma_{1}} \tilde{\tilde{j}}_{\tilde{j}_{1, m_{1}}, p r}^{\varepsilon}\left(t_{1, m_{1}}, L\right) \prod_{m_{2}=1}^{\gamma_{2}} \frac{\tilde{\tilde{j}}_{\tilde{j}_{2, m_{2}}, p r}^{\varepsilon}\left(t_{2, m_{2}}, L\right)}{\left(\gamma_{1}\right)}\right] \\
=\tilde{\mathbb{E}}\left[\prod_{m_{1}=1}^{\gamma_{1}} p_{\tilde{j}_{1, m_{1}}, p r}^{0}\left(t_{1, m_{1}}, L\right) \prod_{m_{2}=1}^{\gamma_{2}} \overline{p_{\tilde{j}_{2, m_{2}}, p r}^{0}\left(t_{2, m_{2}}, L\right)}\right] .
\end{aligned}
$$




\section{Conclusion}

In this paper we have described effects random perturbations of the propagation medium that exhibit long-range correlations have on wave propagation. In Theorem 2.1 and Theorem 2.2 we have considered the case of monochromatic waves, while in Theorem 3.1 and Theorem 3.2 we have investigated the cases of broadband and narrowband pulses. In all these cases the wave propagation is affected by a random mode-dependent and frequency-dependent phase modulation driven by the same fractional Brownian motion for all the propagating modes, and without affecting its energy. Finally, in Theorem 4.1 we have investigated the case of a single-mode waveguide. In this case, the wave propagation is affected by a random time shift given by a fractional Brownian motion without affecting the pulse shape nor its energy. In the notation of Theorem 2.1 the phase modulation appears at depths $L / \varepsilon^{s}$ with $s=1 /(2 H)$ for $H$ the Hurst exponent characterizing the random medium. All these results are in contrast with the mixing case for which all the stochastic effects appear for larger propagation distance $\left(L / \varepsilon^{s}\right.$ with $\left.s=1\right)$. However, the reason why the energy is not affect for the propagation distance $L / \varepsilon^{s}$ with $s=1 /(2 H)$ is that, as already observed for one-dimensional propagation media and the random Schrödinger equation $[11,15]$, the wave does not propagate enough to accumulate enough scattering events to affect its energy.

\section{Acknowledgment}

K. Sølna is partly supported by AFOSR grant \# FA9550-14-1-0197. The work of C. Gomez is carried out in the framework of the Labex Archimède (ANR-11-LABX-0033) and of the A*MIDEX project (ANR11-IDEX-0001-02), funded by the "Investissements d'Avenir" French Government program managed by the French National Research Agency (ANR).

\section{References}

[1] N. I. Akhiezer, The Classical Moment Problem and Some Related Questions in Analysis, Oliver \& Boyd, Edinburgh and London, 1965.

[2] R. Alonso, L. Borcea, And J. Garnier, Wave propagation in waveguides with random boundaries, Commun. Math. Sci., 11 (2012), pp. 233-267.

[3] G. Bal, T. Komorowski, L. Ryzhik, Asymptotics of the phase of the solutions of the random Schrödinger equation, Arch. Rat. Mech. Anal., 100 (2011), pp. 613-664.

[4] P. Billingsley, Convergence of probability measure, $2^{\text {nd }}$ ed., Wiley InterScience, 1999.

[5] L. Borcea, L. Issa, And C. Tsogka, Source localization in random acoustic waveguides, SIAM Multiscale Model. Simul., 8 (2010), pp. 1981-2022.

[6] S. Dolan, C. Bean, And B. Riollet, The broad-band fractal nature of heterogeneity in the upper crust from petrophysical logs, Geophys. J. Int., 132 (1998), pp. 489-507.

[7] A. C. Fannjiang And K. Sølna, Propagation and time-reversal of wave beams in atmospheric turbulence, SIAM Journal on Multiscale Model. Simul., 3 (2005), pp. 522-558.

[8] A. C. FAnNJiAng AND K. Sølna, Scaling limits for beam wave propagation in atmospheric turbulence, Stoch. Dyn., 4 (2004), pp. 135-151.

[9] J.-P. Fouque, J. Garnier, G. Papanicolaou, and K. Sølna, Wave propagation and time reversal in randomly layered media, Springer, New York, 2007.

[10] J. GARNIER, The role of evanescent modes in randomly perturbed single-mode waveguides, Discrete and Continuous Dynamical Systems-Series B, 8 (2007), pp. 455-472.

[11] J. Garnier AND K. Sølna, Pulse propagation in random media with long-range correlation, SIAM Multiscale Model. Simul., 7 (2009), pp. 1302-1324.

[12] C. Gomez, Wave propagation and time reversal in random waveguides, Ph.D dissertation, Université Paris-Diderot (Paris7), 2009. http://tel.archives-ouvertes.fr/tel-00439576/fr/. 
[13] C. Gomez, Wave propagation in shallow-water acoustic random waveguides, Commun. Math. Sci., 9 (2011), pp. 81-125.

[14] C. Gomez, Radiative Transport Limit for the Random Schrödinger Equation with Long-Range Correlations, J. Math. Pures. Appl., 98 (2012), pp. 295-327.

[15] C. Gomez, Wave Decoherence for the Random Schrödinger Equation with Long-Range Correlations. Commun. Math. Phys., 320 (2013), pp 37-71

[16] S. Holm And R. Sinkus, A Unifying Fractional Wave Equation for Compressional and Shear Wave. J. Acoust. Soc. Am., 127 (2010), pp. 542-548.

[17] R. Marty And K. Sølna, Acoustic waves in long range random media SIAM J. Appl. Math., 69 (2009), pp. 1065-1083.

[18] R. Marty And K. Sølna, A general framework for waves in random media with long-range correlations, Ann. Appl. Probab, 21 (2011), pp. 115-139.

[19] G. C. Papanicolaou And K. Sølna, Wavelet based estimation of Kolmogorov turbulence, In Long-range Dependence: Theory and Applications, P. Doukhan, G. Oppenmeim and M. S. Taqqu, editors, Birkhauser, (2002), pp. 473-507.

[20] C. Sidi And F. Dalaudier, Turbulence in the stratified atmosphere: Recent theoretical developments and experimental results, Adv. in Space Res., 10 (1990), pp. 25-36.

[21] K. Sølna, Acoustic pulse spreading in a random fractal, SIAM J. Appl. Math., 63 (2003), pp. 1764-1788.

[22] M. S. TAqqu, Law of the Iterated Logarithm for Sums of Nonlinear Functions of Gaussian Variables that exhibit long range dependence, Z. Wahrscheinlichkeistheorie, 40 (1977), pp. 203-238. 